\title{
Challenges in computational modeling of two-phase transport in polymer electrolyte fuel cells flow channels: a review
}

\author{
Alex Jarauta ${ }^{1}$ - Pavel Ryzhakov ${ }^{2}$
}

Received: date / Accepted: date

\begin{abstract}
Excess of liquid water in gas channels of polymer electrolyte fuel cells is responsible for malfunctioning of these devices. Not only it decreases their efficiency via partial blockage of reactants and pressure drop, but it can also lead to the irreversible damage due to oxygen starvation in case of complete channel flooding or full coverage of the gas diffusion layer with a liquid film. Liquid water evacuation is carried out via airflow in gas channels. Several experimental and computational techniques have been applied to date for the analysis of the coupled airflow-water behavior in order to understand the impact of fuel cell design and operation regimes upon the liquid water accumulation. Considerable progress has been achieved with the development of sophisticated computational fluid dynamics (CFD) tools. Nevertheless, the complexity of the problem under consideration leaves several issues unresolved.

In this paper, analysis techniques applied to liquid water-airflow transport in fuel cells gas channels are reviewed and most important results are summarized. Computationally efficient, yet strongly simplified analytical models are discussed. Afterwards, CFD approaches including the conventional fixed grid (Eulerian) and the novel embedded Eulerian-Lagrangian models are described. Critical comparative assessment of the existing methods is provided at the end of the paper and the unresolved issues are highlighted.
\end{abstract}

\footnotetext{
${ }^{1}$ A. Jarauta

Energy Systems Design Lab (ESDLab), University of Alberta, Edmonton

${ }^{2}$ P. Ryzhakov, corresponding author Centre Internacional de Mètodes Numèrics en Enginyeria Campus Nord UPC, C1, c.Gran Capità, s/n, 08034 Barcelona E-mail: pryzhakov@cimne.upc.edu
}

Keywords PEFC · PEM fuel cell · water management · liquid transport $\cdot$ gas channels $\cdot$ multi-phase . CFD

\section{Introduction}

Polymer electrolyte fuel cells (PEFCs) are one of the leading candidates to replace internal combustion engines for vehicles, as they are the only presently available technology that can offer equivalent power density, range, and refueling times in a sustainable way. PEFCs fueled with hydrogen are environmentally friendly and their efficiency is up to 3 times higher than that of high-temperature combustion devices 38, 93. Hydrogen produced by water electrolysis using excess renewable energy could then lead to emission-free transportation section. The commitment of the industries to fuel cell technology is reflected in the small-scale production of fuel cell vehicles, such as Toyota Mirai and Hyundai ix35 Fuel Cell. Nevertheless, engineering factors such as high cost and limited durability still preclude the PEFC from large-scale commercialization. Operating principle of a PEFC is presented next and the critical factors responsible for its malfunctioning are specified.

Operating principle of PEFCs Both the fuel (hydrogen - on the anode side) and the oxidant (oxygen or air - on the cathode side) are fed to the fuel cell in the gaseous state through the gas channels (see Fig. 1). The gases on both the anode and the cathode side diffuse through the gas diffusion layers (GDLs) towards the reaction site. The reaction takes place when the fuel and oxidant molecules reach the catalyst layers (CLs). The CL is produced using a platinum-based ink which is painted on either the membrane or the GDL. The 
ink contains carbon, $\mathrm{Pt}$ and electrolyte. The resulting coating is a thin (between 1-20 $\mu \mathrm{m}$ ) porous layer.

On the anode, once the hydrogen molecules reach the CL, Pt catalyzes the first reaction, i.e., the hydrogen oxidation reaction (HOR):

$\mathrm{H}_{2} \rightarrow 2 \mathrm{H}^{+}+2 \mathrm{e}^{-}$

After the hydrogen atoms are split into ions and electrons, the polymer electrolyte membrane ensures the conductivity of hydrogen protons, but remains practically impermeable for the electrons in order to send the current through the external circuit.

Electrons travel in the opposite direction through the GDL and the current collector (that at the same time act as the walls of the anode gas flow channel) in order to meet the protons at the other side of the membrane, thus generating the desired electric current.

On the cathode side, oxygen is fed through the gas channels to diffuse through the GDL and react at the catalyst layer with the protons created by the oxidation reaction at the anode. Thus the oxygen reduction reaction $(\mathrm{ORR})$ takes place:

$2 \mathrm{H}^{+}+2 \mathrm{e}^{-}+\frac{1}{2} \mathrm{O}_{2} \rightarrow \mathrm{H}_{2} \mathrm{O}$

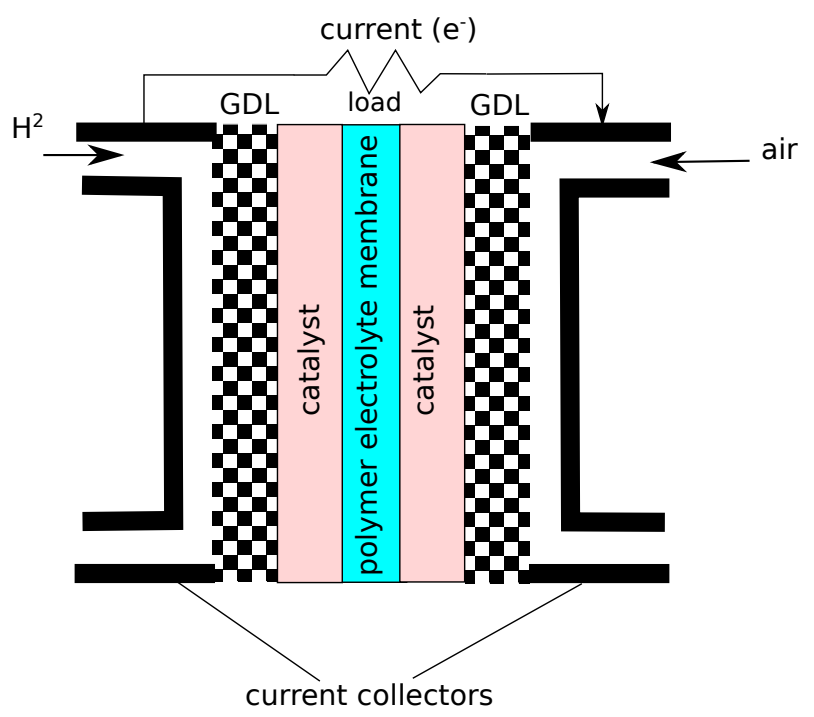

Fig. 1 Schematic view of a polymer electrolyte fuel cell

The performance of a fuel cell is usually represented by a polarization curve (see Fig. 2) showing the voltage losses against the current density in the cell. The polarization curve is obtained by varying the external load resistance from 0 to $\infty \Omega[13$.

In case of an open circuit there is no current. In this case the voltage is a direct measure of the difference in chemical activity of hydrogen at the anode and cathode.
As the external resistance decreases, an electron current flows through the external circuit, balanced by an ion current through the electrolytic membrane. The steep initial decrease is attributed to the barrier for the electron transfer reactions occurring at the electrodes. This is known as kinetic or activation losses region. With further decrease in resistance, one observes an interval where the voltage decreases almost linearly with the current. This is referred to as the ohmic polarization region. In this region the electrical current is limited by the ionic resistance of the electrolyte material. As the external resistance is decreased further, the current reaches a limiting value where the mass transfer of reactants to the electrode/electrolyte interface limits the reaction. This is known as the mass transfer polarization region (Fig. 2).

Summarizing, while kinetic and ohmic dominate at low and medium current densities, at high currents the losses due to mass transport become the key factor 114 . Reducing mass transport losses at high current density would permit fuel cells to be operated at high voltage and high current thereby enabling an increase of specific power density, reducing the size of fuel cells and, thereby, their cost.

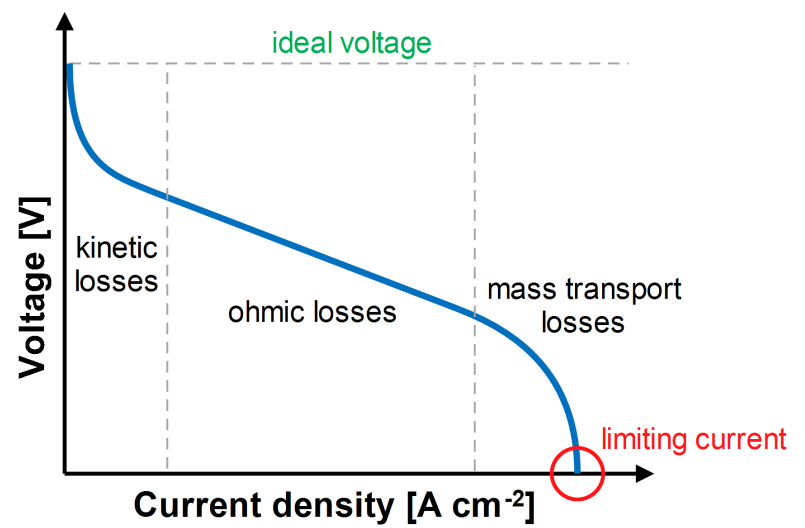

Fig. 2 Polarization curve of a PEFC

Mass transport losses take place when the capability of reactants to reach the catalyst is diminished. Typically, the problem is associated with water accumulation on the cathode side of the cell. The byproduct water must partially humidify the membrane in order to ensure the good ionic conductivity. The minimum volume fraction of water to maintain ionic conductivity is around $6 \%$. Lower amount of water leads to membrane drying. However, excessive water (volume fraction larger than $80 \%$ ) leads to flooding and must be transported through the GDL via capillary-driven flow and evacuated in the gas channels. Liquid water ac- 
cumulation in GDL decreases the effective diffusivity of the layer and reduces the transport of the reactant gases (oxygen, in case of cathode) to the CL. Excess of water in channels leads to non-uniform gas distribution and large pressure losses. Complete flooding in the gas channel or coverage of the GDL leads to oxygen starvation, which can result in an irreversible cell damage due to peroxide formation at the membrane. Thus, the liquid water transport is not only responsible for reducing the cell's efficiency at high current density, but is often the limiting factor to their durability. Therefore, efficient evacuation of liquid water in the gas channels must be ensured. The process of detecting and controlling water distribution in the fuel cell is known as water management.

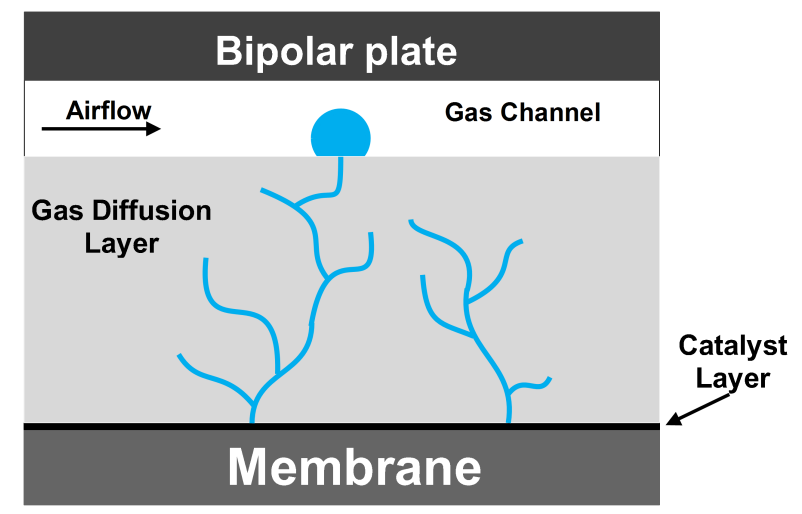

Fig. 3 Schematic view of a droplet emerging from the GDL into flow channel

Experimental observations In order to gain the knowledge of water evacuation characteristics in the fuel cell, one must consider the liquid phase emerging from pores of GDL into gas channel and its subsequent behavior due to the interaction with an airflow (see Fig. 3). Experimental observations have revealed important insights regarding these phenomena (see e.g. [9] for the corresponding review). Apart from being of substantial importance per se, these provide the basis for the validation of computational models.

One can distinguish two classes of experimental techniques: in-situ and ex-situ. The in-situ experiments employ sophisticated technologies, such as neutron imaging [50, 113], X-ray tomography 37, 85, 92], magnetic resonance imaging (MRI) [124, and infrared (IR) thermography 29, 47]. These approaches allow to analyze real fuel cell during their operation. Among other information, they provide valuable data of water distribution in diffusion media and channels, but they rely upon expensive equipment and are difficult to perform.
Also, advanced techniques such as neutron or magnetic resonance tomography require fuel cells designs and/or materials different from those of the real fuel cells.

On the other hand, ex-situ experiments are direct optical observations carried out using a transparent window 17, 28, 120, 121, 130, 132, 134. Although ex-situ studies cannot reproduce an actual fuel cell channel, an immediate visualization access to the channel is granted, enabling the caption of high resolution images. These studies can provide important data for further understanding of wetting phenomena on porous substrates, however it is nearly impossible to reproduce exactly the same settings as the ones found in the real cells. In particular, materials used for transparent inspection windows in ex-situ experiments differ from those of the actual fuel cells in terms of thermal, electrical and microstructural mechanics (wetting characteristics, surface roughness).

Experiments revealed that generally, three types of two-phase flow regimes in gas channels can be distinguished:

\section{- Droplet flow \\ - Film flow \\ - Slug flow}

These flow regimes are depicted schematically in Fig. 4. Droplet flow is commonly observed at low current densities, intermediate current densities typically lead to film flows, and slugs are characteristic of high current densities, defining the most dangerous operation mode from the perspective of the channel blockage. Two key factors in determining the flow regime are the air velocity and the liquid injection rates 55.

Even though experimental observations provide a valuable basis for analysis of two-phase transport in fuel cells, they may present limitations due to the reduced time and length scales of some of these processes. Moreover, the cost of experimental studies precludes their extensive use for testing possible new fuel cells designs and/or different operating conditions.

As a consequence, the researchers have been motivated to develop computer modeling approaches in order to be able to obtain quantitatively valuable information in numerous true operation scenarios and actual fuel cells properties and designs 110 135]. The available experimental data, however, is an extremely valuable material that provides the necessary means for the validation of the computational models.

The majority of the existing computer models belong to one of the following two classes:

- simplified (analytic) models

- continuum two-phase models 


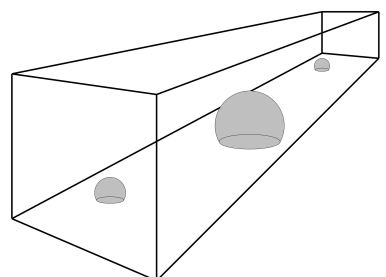

(a) Drop flow

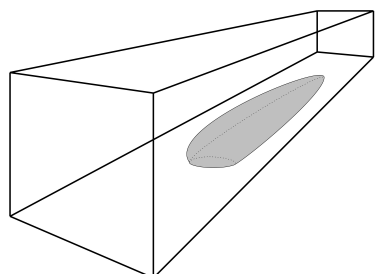

(b) Film flow

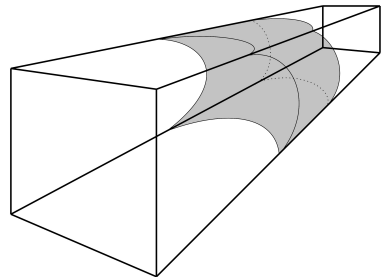

(c) Slug flow

Fig. 4 Flow regimes in PEM fuel cells gas channels

This paper starts by reviewing simplified models (based on macroscopic force balance). Next, continuum based approaches are reviewed. Commonly used volume of fluid (VOF) models are described and their results in application to fuel cell analysis are reviewed. It is emphasized that in spite of the predominantly used fixed-grid VOF method, there exist feasible alternatives based upon using different kinematic frameworks. The possibility of using moving grid approaches is mentioned. Next, a novel embedded technique based on combining fixed grid and moving grid models for gas and liquid phases, respectively, is presented. Several examples comparing the results obtained using different modeling approaches are shown. The paper concludes with the description of open questions and possible future research directions.

\section{Simplified computational models}

Analytic models (also termed "mechanical" or "force balance" models) are based on a simple force balance equation. They are typically restricted to droplet flow regimes as they require an a priori knowledge of approximate liquid phase shape. Rather than explicitly representing the continuum nature of the two phases, analytic models strive to represent the "net" effects. Such models have a low computational cost and are relatively easy to implement.

Chen et al. 18 proposed an analytical model of a water droplet in a PEFC cathode channel based on a macroscopic force balance considering static equilibrium. Other works of liquid droplets dynamics in solid surfaces were published beforehand, but the mentioned study was the first one focusing on a water droplet on a porous surface subjected to an airflow. This work used a force balance equation considering equilibrium shape of a droplet, thus being able to establish a relationship between the surface tension force and the external forces acting on the droplet, i.e. pressure, shear and viscous forces along the channel $z$-direction in a $2 \mathrm{~mm}$ height channel:

$\left(p_{0}-p_{L}\right) 2 B l+l^{2} \tau_{x z}^{w}+f_{\mu, d}=0$

where $p_{0}$ and $p_{L}$ are the gas pressure upstream and downstream of the droplet, respectively, $2 B$ is the height of the channel, $l$ is the width of the channel in the crosschannel direction, $l^{2} \tau_{x z}^{w}$ is the shear stress acting on the droplet, and $f_{\mu, d}$ is the viscous drag. Eq. (3) was derived considering that the droplet had a spherical shape. The force balance resulted in a single equation relating the flow conditions, i.e. mean velocity and air viscosity, and the droplet geometry, namely height, length and contact angle hysteresis. These were expressed as:

$$
\begin{aligned}
& y \sqrt{1-y^{2}}-\left(\cot \theta_{S}\right) y^{2}-\frac{12 \mu U}{\pi \gamma \sin ^{2} \theta_{S}} \\
& \frac{\hat{H}(1+\hat{H})}{\left(1-\cos \theta_{S}\right)(1-\hat{H})^{3} \frac{4 B}{L} \hat{H}\left[1-(1-\hat{H})^{3}\right]}=0
\end{aligned}
$$

where $y=\sin \frac{1}{2}\left(\theta_{A}-\theta_{R}\right), \theta_{S}$ is the static contact angle, $\mu$ is the dynamic viscosity of the gas, $U$ is the average velocity along the channel direction, $\gamma$ is the surface tension coefficient, $\hat{H}$ is the dimensionless droplet height, and $L$ is the channel length. The equation was solved for $y$, and then the contact angle hysteresis was obtained as $\theta_{A}-\theta_{R}=2 \sin ^{-1} y$, where $\theta_{A}$ and $\theta_{R}$ represent the advancing and receding contact angles, respectively. A similar equation was derived considering that the droplet was cylindrical instead of spherical. Note that a steady-state flow regime was considered.

Chen et al. also performed experiments to validate their model. For two different airflow velocities, they measured the contact angle hysteresis at the onset of detachment for different droplet heights. They compared these values to the values predicted by Eq. (4), obtaining a stability window depending on the droplet size. The comparison between the experimental observations and the predicted ones is depicted in Fig. 5 .

The study of Chen et al. 18 concluded that increasing the channel length while maintaining the other parameters, such as GDL hydrophobicity or gas velocity, enlarged the instability window (the angle hystere- 


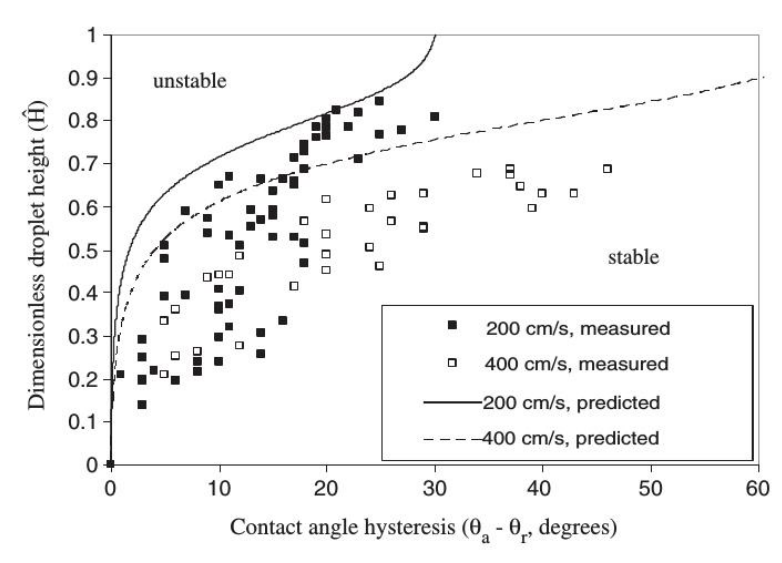

Fig. 5 Effect of airflow velocity on droplet stability. Reproduced from 18] with kind permission of the copyright owner

sis that promotes droplet detachment). The same effect could be achieved if the mean gas flow velocity was increased, which meant that the pressure drop in the channel was higher. By increasing the static contact angle, or equivalently, making the GDL/gas channel interface more hydrophobic, water droplets could be prevented from lodging in the channel. The main drawback of that model was that it did not take into account the effect of pinning (i.e., the contact line between the droplet and the GDL surface was considered to be always circular). One must keep in mind that droplet detachment depends on contact line deformation (see e.g. 62 ). Additionally, area coverage of the water in the GDL surface was not included, and the two-way liquid-gas interaction was not taken into account.

Another analytical model for droplet dynamics in PEFC channels was developed by Kumbur et al. 74. Following an approach similar to that of [18], the droplet geometry was expressed as a function of channel height, GDL contact angle and airflow velocity.

$$
\begin{aligned}
& \frac{24 \mu B^{2} U h^{2}}{(B-h / 2)^{3}\left(1-\cos \left(\theta_{A}\right)\right)^{2}}+\frac{12 \mu B U h^{2}}{(B-h / 2)^{2}\left(1-\cos \left(\theta_{A}\right)\right)^{2}}- \\
& \gamma c \frac{\pi}{2}\left[\frac{\sin \left(\Delta-\theta_{A}\right)-\sin \left(\theta_{A}\right)}{\Delta-\pi}+\frac{\sin \left(\Delta-\theta_{A}\right)-\sin \left(\theta_{A}\right)}{\Delta+\pi}\right]=0
\end{aligned}
$$

where $h$ is the droplet height, $c$ is the droplet's chord length, and $\Delta=\theta_{A}-\theta_{R}$ is the contact angle hysteresis.

Several experiments were also performed in order to observe the airflow conditions that lead to detachment depending on droplet deformation. Fig. 6 displays the experimental results, as well as the detachment predicted by Eq. (5). Similar to the experimental study of Chen et al. [18], two stability zones were identified. Good agreement was found between critical values pre- dicted by their analytical model and the experimental observations.

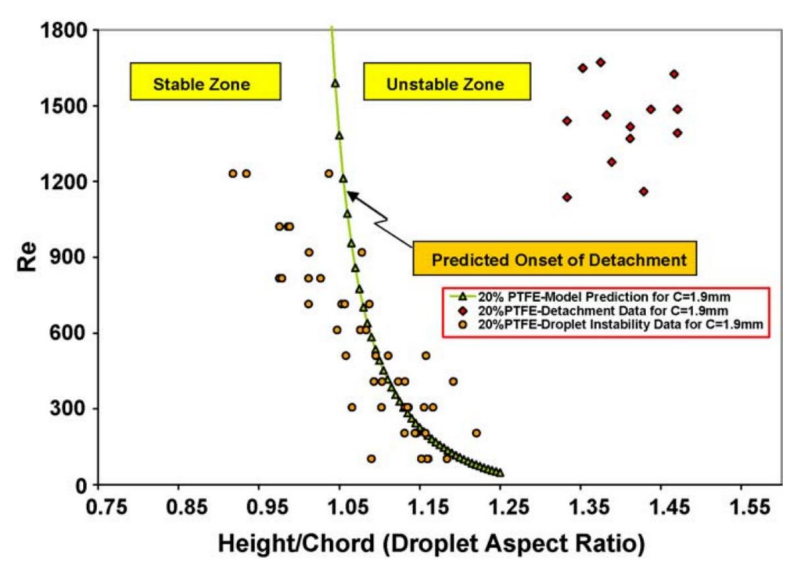

Fig. 6 Effect of airflow velocity on droplet stability. Reproduced from 74 with kind permission of the copyright owner

The experimental data was also used to find a relation between the content of Teflon in the GDL surface and the surface tension. Thus, they improved Chen's work by relating the contact angle hysteresis with the flow conditions, the Teflon content in the GDL and the roughness of the surface, in addition to several geometric variables. Nevertheless, this model could still not account for geometry of the deformed droplet, drag coefficient, area coverage and roughness effects of the GDL. An important conclusion was that at low air velocities, the droplet instability was insensitive to the hydrophobicity of the surface. That fact could be used to minimize costs in the PEFC manufacture and avoid efficiency loss, since a highly hydrophobic GDL has more electrical resistance and is more expensive [74. The analytical equations in this study were also based on the equilibrium of forces, i.e. it was a steady-state analysis.

Esposito et al. 39] proposed a geometry model for a deformed droplet, which was experimentally validated in 96. While the study of Kumbur et al. gave an analytical expression for the hysteresis angle, it did not characterize the main geometric variables of the static and deformed droplet. This study was an improvement compared with the two previous works since the analytical model was transient. Their governing equation was Newton's Second Law applied in one dimension:

$m \frac{\mathrm{d} x_{C M}}{\mathrm{~d} t}=-\gamma \frac{\partial S}{\partial x_{C M}}-\frac{1}{2} \rho U^{2} C_{D} R^{2}\left(\theta_{S}-\frac{\sin \left(2 \theta_{S}\right)}{2}\right)(6)$

where $m$ is the droplet's mass, $x_{C M}$ is the $x$-coordinate of its center of mass, $t$ is time, $S$ is the surface of the droplet in contact with air, $\rho$ is the density of air, $u$ is the mean velocity of airflow, $C_{D}$ is the drag coefficient of 
the droplet, and $R$ is the droplet radius. The first term on the right-hand side of Eq. (6) represents the surface tension force, whereas the second term represents the drag force exerted by the airflow.

Several geometric properties, such as advancing angle, droplet radius, height and perimeter, were expressed as a function of the center of mass x-coordinate $\left(x_{C M}\right)$ for a fixed droplet volume. Although the wetting area over the GDL was approximated by a cylinder, results provided valuable information regarding the studied phenomena.

For instance, the evolution of both advancing and receding contact angles over time could be modeled, as displayed in Fig. 7. The difference between both contact angles is the contact angle hysteresis and is a measure of the droplet's deformation. It can be observed that the droplet deformed over time, reaching a maximum contact angle hysteresis of $100 \mathrm{deg}$ at the end of the simulation.

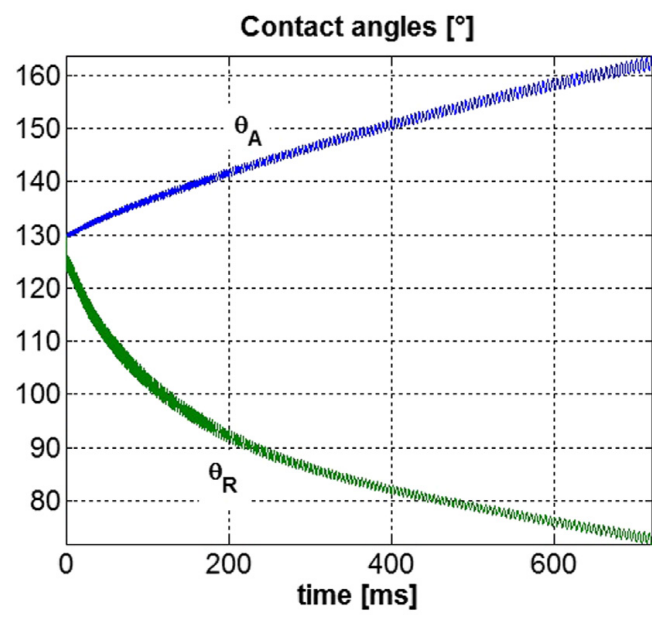

Fig. 7 Evolution of advancing and receding contact angles over time. Reproduced from 96 with kind permission of the copyright owner

In their simulations, Esposito et al. reported that the drag force increased linearly with the increase of $x_{C M}$, whereas the force exerted by the surface tension and the inertial force had a linear decay. On the other hand, $x_{C M}$ was found to decrease over time due to the deformation of the droplet. The transient model as a function of the droplet height proved to be valid for height over $0.5 \mathrm{~mm}$, but the detachment height predictions did not agree with the results of the experiments carried out by the same group. One possible reason is that the drag coefficient used was that from a free spherical particle immersed in a fluid [39]. Therefore, an improvement on this coefficient would probably result in a more accurate model. Furthermore, the models developed by Kumbur et al. and Esposito et al. do not take into account the gravity force since they impose a force balance exclusively in the $x$ direction.

The analytical model developed by Cho et al. 22 considered droplets that were initially spherical and their deformed shape was close to a sphere, so the average curvature could be approximated by the initial radius. This model was a modification of their former model presented in [18]:

$$
\begin{aligned}
& y \sqrt{1-y^{2}}-\left(\cot \theta_{S}\right) y^{2}-R e^{-1} W e_{r} \frac{H}{r} \frac{12}{\pi \sin ^{2} \theta_{S}} \\
& \frac{\hat{H}(1+\hat{H})}{\left(1-\cos \theta_{S}\right)(1-\hat{H})^{3}(4 B / L) \hat{H}\left[1-(1-\hat{H})^{3}\right]}=0
\end{aligned}
$$

where $R e$ is the Reynolds number and $W e_{r}$ is the Weber number. The authors took the results of Chen et al. 18 as a starting point but excluded the hypothesis of the droplet constant shape. Their results showed that at low gas velocities, the viscous force proved to be dominant on small droplets whereas on large droplets the normal force was dominant. On the other hand, the droplet deformation increased significantly when the droplet height was close to the channel height, as shown in Fig. 8.

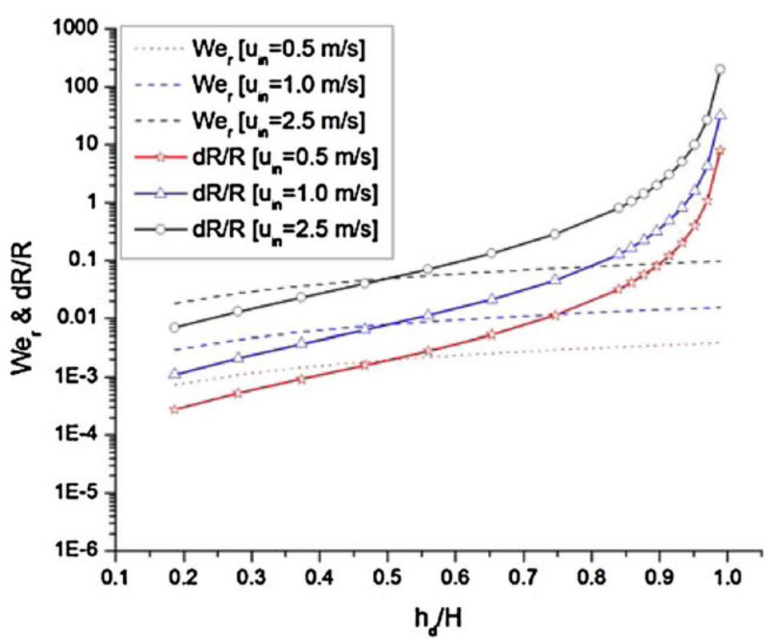

Fig. 8 Deformation of a droplet as a function to the droplet height-to channel height ratio. Reproduced from 22 with kind permission of the copyright owner

The detachment velocity was analyzed using a generalized equation for the drag coefficient $C_{D}$ obtained with fitted data for the conditions of the study. As expected, the detachment velocity decayed with an increasing droplet diameter, and the relationship between 
the Weber and the Reynolds numbers found in reference 18 was corrected. In their model, Cho et al. 22 did not describe the geometry of the deformed droplet, contact line deformation, or roughness effects of the GDL on droplet dynamics.

Jarauta et al. 62 proposed an improved semi-analytic model extending the approach of Esposito [39. The governing equation was again Newton's Second Law applied to the $x$-coordinate of the droplet's center of mass. However, the adhesion force was obtained using the formula proposed by Extrand et al. [40], which could be used to obtain the adhesion force for any contact line geometry:

$F_{\text {adh }}=-\gamma \int_{0}^{l} \cos (\theta(l)) \cos (\psi(l)) d l$

where $\theta$ is the contact angle at the azimuthal position $l$ of the contact line, and $\psi$ is the projection of surface tension force onto the $x y$ plane. For the drag force, an expression for the drag coefficient, $C_{\mathrm{D}}$, based on CFD simulations considering several droplet geometries under study was used (thus, the model was entitled semianalytical). The variables selected to include in the drag coefficient equation were the Reynolds number $R_{h}$ (using the droplet's height as the characteristic length) and the contact angle hysteresis, $\Delta$, of the droplet (as a measure of its deformation). Two different scenarios were identified: for droplets with height less than $10 \%$ of the channel height, the relationship between $C_{D}$ and the variables $R e_{h}$ and $\Delta$ was linear. For droplets with a height greater than $10 \%$ of the channel height, the relationship became non-linear. A fitted equation for both cases was provided.

The semi-analytical model was validated by comparing with experimental data available in literature. In this case, results were compared to those from Milne et al. [89], which measured the critical drag force needed to detach a sessile droplet from a hydrophobic surface.

Fig. 9 shows the comparison between the experimental results of 89 , the predicted values with the proposed model, and the values obtained with the spring model in reference 39 . The values predicted with the semi-analytical model were in good agreement with the experimental data. The values obtained with a spring model largely underpredict the critical drag force.

Summarizing, all the above-described models can be used to predict the detachment on-set conditions and/or detachment times for droplet flow regime. The most common onset criterion is based on the critical contact angle hysteresis. This value characterizes given GDL material and is obtained from experiments. Thus, the analytical (and semi-analytical) models allow to identify the conditions leading to this value. However,

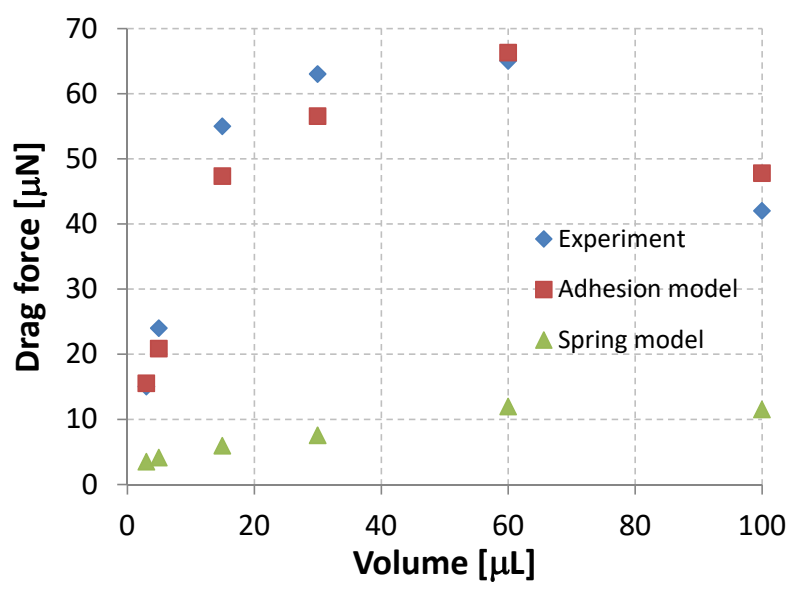

Fig. 9 Validation results of the semi-analytical model, reproduced from 62 .

these models are limited to single droplets with predefined geometries and smooth GDL surfaces.

\section{CFD models}

Although models based on macroscopic force balance provide solutions at low computational cost, they oversimplify the analyzed phenomena either by assuming known droplet geometry or neglecting airflow-liquid interactions. Thus for the cases characterized by strong deformation of the liquid domain, taking place in case of gravity dominated flows leading to film or slug flow patterns, analytical models cannot be applied. Even for droplet flow, the difference between experimental and analytical predictions can be as high as $30 \%$ for highly deformed droplets.

Two-phase CFD approaches that are explicitly modeling both phases involved in the problem under consideration and are equipped with the means for liquid-gas interface tracking define the class of approaches that can provide valuable insights regarding the flow patterns and liquid water evacuation for, theoretically, arbitrary flow conditions. Even though there exist several well-established numerical strategies for solving the two-phase transport problems with moving interfaces, the particularity of the liquid-airflow interaction in the fuel cells gas channels defines a very challenging setting for the general-purpose CFD approaches.

The flow conditions found in a fuel cell's flow channel are rather different from those encountered in many classical engineering applications that involve coupled liquid-gas flow. In a fuel cell one deals with sub-millimeter hydraulic diameter of the channel, presence of various length and time scales and complex wetting characteristics of the solid material (channel walls and GDL sur- 
face). The liquid flow is laminar (Reynolds number less than 10) and is characterized by the dominant effect of the surface tension force. Dimensionless numbers relating surface tension to other forces are (a) capillary number, $\mathrm{Ca}$, which is the ratio between viscous and surface tension forces, (b) bond number, Bo, which is the ratio between gravitational and surface tension forces, and (c) Weber number, We, which is the ratio between inertial and surface tension forces. For droplets in microchannels, Ca $10^{-5}, \mathrm{Bo} \sim 10^{-2}$, and We $10^{-4}$.59. Although surface tension effects are dominant, the rest of forces cannot be neglected making transient modeling obligatory. For problems with dominant surface tension effects many of the well-established computational strategies for solution of the conservation equation of the continuum result in excessive computational times or even fail 88,108 . On the other hand, airflow is characterized by Re between 1000 and 5000, and viscous and convective effects are dominant in this domain.

CFD models are typically based on the incompressible Navier-Stokes equations for two-phase flows. The challenges to be faced in these models can be categorized into two types. First are the general challenges of multi-phase models. These include:

- Detecting the interface between the phases

- Accounting for the change in fluid properties across the interface

- Modeling the flow variables discontinuity across the interface

- Modeling the interaction between the phases (coupling)

The second type are the challenges specific to the problem of interest. These appear due to microscopic nature of the flow and complex solid media. These are:

- Inclusion of surface tension effects in an efficient and robust way

- Accounting for contact with solid phase characterized by complex wetting properties (GDL/channel interface)

- Accounting for presence of different length and time scales (injection from the tiny GDL pores, flow in gas channels)

The way these challenges are tackled strongly depends on the overall kinematic framework adopted for the problem under consideration. Fixed-grid and moving grid approaches can be distinguished.

\subsection{Fixed-grid models}

The majority of existing numerical models used for analysis of two-phase flow in fuel cells are based on fixed- grid (Eulerian) kinematic framework for the description of the two-phase problem 66, 101, 103, 115, 120 . 132,138. They rely on solving the Navier-Stokes equations for both phases on a unique fixed mesh using a special technique for determining the interface between the gas and the liquid 1 . This interface is usually captured by the VOF metho 2 originally proposed by Hirt and Nichols [51]. In what follows, "Eulerian approaches equipped with VOF interface capturing technique", will be simply referred to as "VOF models" following the generally accepted abuse of the terminology. In contrast with the early continuum-based mixture or mist models 87,123 , one deals here with a truly two-phase model where the geometry of the deforming interface is represented.

\subsubsection{Rationale of VOF models}

According to the VOF method, a volume fraction of the fluid $k, C_{k}$ is computed at each element of the computational domain as:

$-C_{k}(\mathbf{x}, t)=0$ outside fluid $\mathrm{k}$

$-C_{k}(\mathbf{x}, t)=1$ inside fluid $\mathrm{k}$

$-0<C_{k}(\mathbf{x}, t)<1$ at the interface

The sum of volume fractions in each element is equal to 1 . The evolution of the volume fraction is governed by the advection equation:

$\frac{\partial}{\partial t}\left(C_{k} \rho_{k}\right)+\nabla \cdot\left(C_{k} \rho_{k} \mathbf{v}_{k}\right)=0$

where $\mathbf{v}$ is the velocity vector.

Considering both air and water as Newtonian incompressible fluids, the governing equations in the microchannel are given by the Navier-Stokes equations equipped with the incompressibility condition:

$$
\begin{aligned}
& \rho \frac{D \mathbf{v}}{D t}-\mu \nabla \cdot\left(\nabla \mathbf{v}+\nabla^{T}(\mathbf{v})\right)+\nabla p=\mathbf{f}_{e x t} \\
& \nabla \cdot \mathbf{v}=0
\end{aligned}
$$

where $\frac{D \mathbf{v}}{D t}$ is the material time derivative of the velocity, $p$ is the pressure, and $\mathbf{f}_{e x t}=\mathbf{f}_{g}+\mathbf{f}_{s t}$ is the external

1 Continuum-based modeling in the PEFCs started with the mixture models (so-called $M^{2}$ models) 95 126 131. Mixture approaches model the liquid-gas system as a single phase with a varying phase composition. Liquid water saturation is treated as a liquid volume fraction. While mixture models can provide valuable information regarding the amount of liquid water and its effect upon the fuel cell performance (particularly at low current densities), they are intrinsically incapable of representing the separate phases and the interface between them and cannot be used for a detailed analysis of liquid water evacuation in gas channels.

2 An alternative to the VOF method is the level set (LS) method 94, 105], however it has been seldom used in the context of two-phase transport modeling in PEFC channels $24 \mid 25$ 
force comprising of gravity and the surface tension. The material properties $\rho$ and $\mu$ are the volume-averaged density and dynamic viscosity computed according to the corresponding volume fractions as:

$\rho=\rho_{g}+C_{l}\left(\rho_{l}-\rho_{g}\right)$

$\mu=\mu_{g}+C_{l}\left(\mu_{l}-\mu_{g}\right)$

where subindices $l$ and $g$ stand for liquid and gas, respectively.

Surface tension is computed according to LaplaceYoung equation:

$\mathbf{f}_{s t}=\Delta p \mathbf{n}=\gamma \kappa \mathbf{n}$

where $\Delta p$ is the pressure jump across the interface, $\mathbf{n}$ is the unit normal to the interface, and $\kappa$ is the interface curvature. It can be shown that this term leads to the following expression:

$\mathbf{f}_{s t}=\gamma \rho \kappa \frac{\nabla C_{k}}{0.5\left(\rho_{l}+\rho_{g}\right)}$

This is known as the continuum surface force (CSF) model for surface tension [15. It is the most used method for surface tension calculation in fixed-grid models [41. The term $\kappa$ in Eq. (15) is the surface curvature, defined as the divergence of the unit normal to the interface as

$\kappa=\nabla \cdot \frac{\mathbf{n}}{\|\mathbf{n}\|}$

The normal is computed as the gradient of the volume fraction $\mathrm{k}$ :

$\mathbf{n}=\nabla C_{k}$

An alternative to CSF model is the continuum surface stress (CSS) model, developed by Lafaurie et al. [75. According to CSS model the surface tension term has the following expression:

$\mathbf{f}_{s t}=\nabla \cdot\left(\gamma\left(\left\|\nabla C_{k}\right\| \mathbf{I}-\frac{\nabla C_{k} \otimes \nabla C_{k}}{\left\|\nabla C_{k}\right\|}\right)\right)$

CSS model has several advantages over CSF model: it is conservative and it does not require the computation of the curvature $\kappa$. Moreover, it can be used to solve problems with variable surface tension, whereas CSF model cannot account for this effect.

At the contact with the solid phase, a contact angle is representing the wetting characteristics of the solid. In VOF contacts with substrates characterized by different hydrophobicity (measured by the contact angle) are modeled according to 15 . Rather than imposing the contact angle directly as a boundary condition, the contact angle characterizing the given liquid-solid system (and typically known from experiments) is used to adjust the normal to the liquid surface in cells near the solid wall. This so-called dynamic boundary condition results in the adjustment of the curvature of the surface in the vicinity of the solid surface and is then used when computing the surface tension term in the cells next to the triple contact (liquid-gas-solid). Thus, it can be viewed as an a-posteriori correction.

If $\theta$ is the contact angle at the wall (i.e. between the wall and the tangent to the phase interface, as shown in Fig. 10, then the surface normal at the cell next to the wall is computed as

$\mathbf{n}=\mathbf{n}_{w} \cos \theta+\mathbf{t}_{w} \sin \theta$

where $\mathbf{n}_{w}$ is the unit normal vector of the wall's surface, and $\mathbf{t}_{w}$ is the wall's unit tangent vector (Fig. 10.

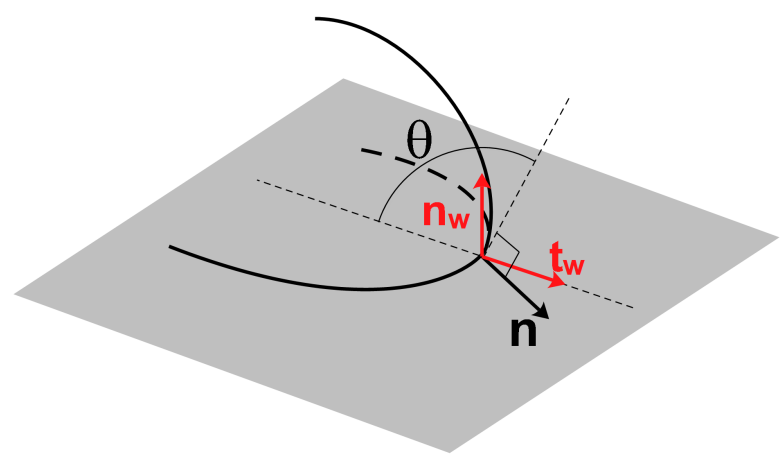

Fig. 10 Unit normal to the droplet's surface and contact angle with respect to the substrate

\subsubsection{VOF modeling results}

Some studies in literature have considered single-phase flow in the channel, such as the work of Lobato et al 81]. However, only models that consider two-phase flow are described here. Generally, one can distinguish between the models considering droplets with fixed volume in the flow channel/GDL interface and the models that strive to reproduce droplet injection. All numerical studies described in this section use the CSF model for surface tension, unless mentioned otherwise.

Earlier studies of droplet dynamics in fuel cell channels include the works by Golpaygan and Ashgriz 43 . 44], and Shirani and Masoomi 115]. The computational domain in these studies was a fraction of a channel with a water droplet in contact with the lower wall. Effects of surface tension, fluid density and viscosity, and gas velocity on droplet deformation and removal were studied. Surface tension was modeled using CSS approach. Results showed that reducing the surface tension coefficient was the most effective approach for promoting 
water removal. However, the contact line of the droplet was fixed, and no validation was provided. Golpaygan and Ashgriz extended their work to 3D analysis [45], and the same conclusions were extracted. Shirani and Masoomi 115 reported a time step of $10^{-5} \mathrm{~s}$ for simulations of $20 \mathrm{~ms}$.

Straight channels with fixed liquid volumes have

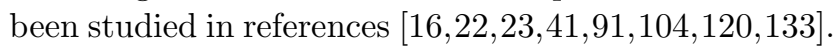
Cai et al. 16] concentrated on the impact of wetting properties of the channel walls and the GDL upon the liquid evacuation. It was concluded that hydrophilic channel side-wall with a hydrophobic GDL surface could avoid water accumulation on the membrane electrode assembly (MEA) surface. Post-detachment behavior of droplets was also investigated. Such setting leads to joining the individual droplets into liquid films accumulated at the channel walls [16], which can be easily drained. A similar study was performed by Zhan et al. 133, and it was also observed that hydrophilic channel walls attracted more water, therefore helping oxygen diffusion into the GDL. In this work both straight and serpentine channels were considered.

The first study that included experimental validation was developed by Theodorakakos et al. 120. In their work, they focused on the detachment of liquid droplets from GDLs under the influence of cross-flowing air. Simulation results were compared with those obtained experimentally with a transparent channel, and good agreement was obtained. Parametric studies investigating the effects of various parameters showed that a) a droplet was easier to remove by a uniform air velocity pattern rather than a developing flow, b) decreasing surface tension values resulted in reduced adhesion force and faster removal (in agreement with Golpaygan 43]), c) gravity effects were negligible, and d) droplets were removed at lower velocities when in contact with the channel walls.

Cho et al. performed a comparison among the experimental data and the simulations using analytical 22] and VOF models 23. Results predicted with analytical model were in good agreement with experimental data, and the study concluded that larger droplets needed less airflow velocity for detachment.

Water transport in U-shaped channels in order to investigate the particularity of serpentine channels has been analyzed in $17,53,63,73,103$. Following the same approach than studies considering straight channels, liquid water was placed in different configurations (droplets, films, etc) in arbitrary locations. Quan et al. 103 focused their study in the bend area. It was shown that the secondary flow appeared due to dropletairflow interaction which affected the liquid behavior in the vicinity of the bend, driving the water to the chan- nel walls. When performing simulations with large liquid volumes, it was shown that the GDL surface (after the bend) may become covered impoverishing the reactants supply. A recent study by Hou et al. 53. analyzed the effects of the bend area shape on the droplet motion along the channel.

Jiao et al. extended the work of Quan et al. [103 by including the inlet and outlet manifolds in two different channel configurations: straight parallel channels 65. and serpentine parallel channels 67. Several airflow problems characteristic for this type of channels were identified and suggestions for improving the water removal were given based on the obtained flow patterns in channels and manifolds. It was concluded that U-bends facilitated water removal as they led to separation of liquid films into small droplets. A recent study by Ashrafi et al. 11 studied droplet deformation and detachment in parallel tapered channels.

All the above-mentioned simulations were performed considering a fixed liquid volume and a uniform GDL. However, experimental observations show that water enters through the pores of the GDL surface and that the emergence, growth and detachment play an important role in the resulting flow regime. Therefore, many numerical studies in literature have analyzed liquid water injection in straight channels $12,19,21,30,33,34$ 49, 72, 97, 136 140.

Zhu et al. analyzed water injection from a GDL pore into a straight channel using two-dimensional numerical simulations 137,140. Fig. 11 shows the evolution of the liquid domain emerging from pores of $50 \mu \mathrm{m}$ and $25 \mu \mathrm{m}$ width.

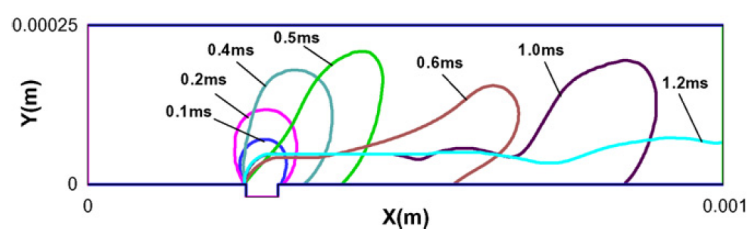

(a) Pore width of $50 \mu \mathrm{m}$

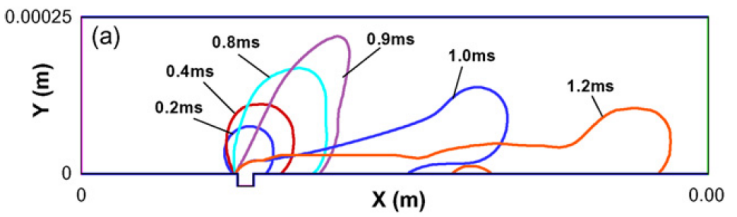

(b) Pore width of $25 \mu \mathrm{m}$

Fig. 11 Evolution of liquid injected from a GDL pore. Airflow velocity $u=10 \mathrm{~m} / \mathrm{s}$, water injection velocity $w=1 \mathrm{~m} / \mathrm{s}$. Reproduced from 137. with kind permission of the copyright owner. 
Effects of the static contact angle, pore size and air and water inlet velocities on the water droplet dynamics were analyzed. As observed in previous studies, a more hydrophobic GDL surface promoted water removal. A hydrophilic surface lead to film pattern formation, thus blocking air diffusion into the GDL. Smaller pore sizes showed a slower rate of both droplet deformation and break-up processes. Results also showed that for both low and high airflow velocities, water droplet formed a film flow. High water injection velocities lead to channel blockage. However, the contact angle on the GDL surface was considered constant, neglecting roughness effects, and contact angle hysteresis was not accounted for. The same authors extended their work into a three-dimensional study in references [136, 138], reporting similar results. Effects of cross-section on water transport were analyzed [136, obtaining an optimal height-to-width ratio of 0.5 for water removal. Effects of pore location on water transport were studied in [139]. When water emerged from the center of the channel, its transport was only a function of GDL contact angle. Channel walls' contact angle was the driving parameter for droplets emerging close to the channel's corners. Zhu et al. 138] reported a time step of $10^{-7} \mathrm{~s}$ for their simulations, with a total simulated time of $5 \mathrm{~ms}$. The reduction in time step is due to the simulation of water injection through the pore, which previous works did not consider.

Bazylak et al. 12 performed numerical simulations of water emerging through 2 interconnected pores. Their simulations were compared to experiments using fluorescence microscopy. Results showed that the water emergence occurs via preferential locations corresponding to the path of least resistance changing in time.

A 3D+1D model was developed by Ding et al. 33. Single-phase and two-phase simulations were performed to identify effects of liquid flow patterns on fuel cell performance. The efficiency of the fuel cell was reduced when slugs were present in the channel. On the other hand, a more hydrophobic GDL surface and higher air velocities improved the performance of the cell. Ding et al. reported a computational time of 2 weeks for their calculations. The previous study was extended by the same authors 34] in order to observe maldistribution of reactant gases in two parallel channels. An uneven distribution of gases led to slug formation, reducing the fuel cell performance. None of the studies presented by Ding et al. 33, 34 considered water transport in the MEA, which is a critical factor for fuel cell performance.

Droplet-droplet interaction was studied by Kim et al. 72 considering a straight channel with two parallel pores. Several variables were considered, such as poreto-pore distance, pore-to-wall distance, wettability of the GDL and airflow velocity. Similar to previous observations, hydrophobic channel walls led to slug formation, and droplet coalescence improve water removal.

Only a few recent works analyzed more complex configurations, considering multiple droplets rather than single isolated drop. Coalescence of droplets and movement of slugs was simulated by Carton et al. [17] considering double serpentine channels. It was concluded that the droplet coalescence may lead to creation of slugs and thus hinder water evacuation. Moreover, after one of the channels was blocked, airflow redistributed to the other channel. Inter-droplet interactions have also been studied in 72 . There, droplets injected from two GDL pores have been analyzed. The authors studied the effect of the distance between the pores as well as the influence of the channel width and operating conditions upon the water removal. It was concluded that a large drop resulting from coalesced drops can be removed with greater facility than two separate droplets. Flooding was likely to occur in case of using hydrophobic channel walls and low air velocity. Alternative studies using more complex geometries are those from Quan and Lai [101, 102, Jiao and Zhou 64, Le et al. [79, Kang et al. [70, Wang and Zhou [125], and Jo and Kim 68]. Novel channel designs and their effects on water transport have been analyzed by Fontana et al. [42, Mancusi et al. 84, and Qin et al. 98 100. Models considering the full cell have been presented by Le and Zhou 76 78, 80.

Studies of two-phase flow in channels provided important insights of the influence of liquid injection rates, hydrophobicity, crossflow velocity, geometry of the channels upon the liquid water evacuation. However, for design purposes it is important to accurately model the injection from the GDL which occurs at multiple locations with different rates. Previously mentioned studies considered hypothetical initial configurations of the droplets in the channels. Moreover, roughness effects of the GDL also strongly impact the detachment behavior of droplets. Thus, coupling of microscopic transport through the GDL and the macroscopic transport in the channel appears to be obligatory for obtaining deeper insights of the overall two-phase transport behavior. Moreover, only this way new generation of accurate and detailed overall fuel cell models can be derived.

Some authors idealized the microstructure by using patterned pores with simplified geometries. He et al. 49 considered triangular and rectangular shapes to represent the GDL substrate. Ding et al. 31, 32, and Hossain et al. 52 reproduced the microstructure of a GDL by means of a circular pore mesh. Recently, Ashrafi and Shams [10] studied effects of GDL roughness in a two-dimensional study, where roughness size 
and distribution was randomly generated for several cases. Chen et al. considered a regular set of cubic indentations on the GDL surface, representing Cassie approximation of surface roughness 1921 . The authors concluded that GDL surface roughness reduces the retentive forces and increases the detaching forces. Nevertheless, validity of representing the non-homogeneous porous surface by a regular imperfection sets remains under question. Moreover, there exist no straight-forward way of estimating the parameters of a regular geometry necessary for representing the "average" properties of a heterogeneous surface. Once again, resolving the pore-scale geometrically is computationally unfeasible, but must be performed obligatorily for validating the simplified models. Time steps reported in these studies were $10^{-7} 1921$ and $2 \times 10^{-7} \mathrm{~s} 49$, with total simulation times of the order of $10^{-2} \mathrm{~s}$.

Few studies that considered GDL structure explicitly appeared in the past several years. $2 \mathrm{D}$ simulations based on reconstruction of fiber-arrangement of actual GDLs have been carried out in $7,8,117$. In [7] latter work the effect of polytetrafluoroethylene (PTFE) loading in the GDL was also studied by varying the corresponding contact angle boundary conditions. The results of the corresponding simulation are shown in Fig. 12. The GDL is represented as a 2D domain consisting of $8 \mu \mathrm{m}$-diameter circles separated by a distance of $18 \mathrm{~m}$ measured from the centers of the circles. Similarly, in 117. GDL is idealized as a perfectly regular structure with the fibers of unique thickness. Thus, once again a hypothetical configuration is considered. Nevertheless, experimental studies show that GDL is a highly heterogeneous structure and the experiments as well as pore-network simulations reveal that the location of preferential sites of liquid emergence strongly depends on this heterogeneity 12,46 .

Possibly, the dominant application of VOF model to analysis of two-phase flow in fuel cell can be explained by the availability of this model in the commercial CFD software packages. Except for the works of Golpaygan and Ashgriz 43 45], Shirani and Masoomi 115], and Theodorakakos et al. 120], the rest of the above-mentioned references use ANSYS Fluent [2] for their numerical analysis 41. In all these studies, the explicit CSF model has been used for surface tension. Therefore, the time step used has been limited due to the time scale associated with the propagation of capillary waves $[15$.

The time step restriction makes the use of the models based on explicit integration of surface tension computationally tedious. Their application to transient twophase simulations in several components (diffusion media and gas channel), which is necessary for obtaining

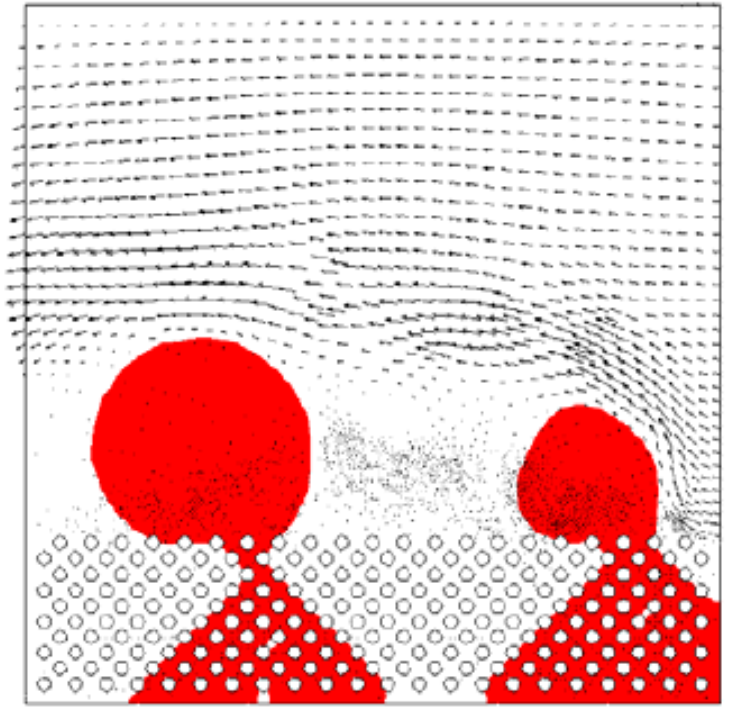

Fig. 12 Contour plot of water droplet forming on top of GDL surface with $15 \%$ PTFE loading and deforming due to air flowing in the channel. Reproduced from [7] with kind permission of the copyright owner

quantitative predictions for water management, can be therefore rather limited. Moreover, due to the absence of interface meshes, these approaches model surface tension as a continuous body force spreading across a transition region of finite thickness, which may lead to nonphysical velocities in the vicinity of the interface [57].

The mentioned approaches are often relying on prescribing static contact angles and are incapable to represent the contact dynamics and pinning effects, essential taking into account rough GDL surfaces. Fixed grid approaches also exhibit deficiencies in dealing with topological changes such as droplet breakup and coalescence [128]. If two droplet boundaries appear in the same mesh element, they automatically merge leading to spurious numerical coalescence.

Summarizing, one can identify the following features missing in the majority of VOF-based studies applied to analyze two-phase transport in fuel cells to date:

- Implicit formulation of surface tension term. Explicit integration results in severe restrictions for the stable time step size.

- Representation of surface tension as a surface force. Its modeling as a volume force may manifest in spurious movements of the interface.

- Efficient coupling of the transport through different components of the cell.

- Accurate representation of wetting dynamics on the GDL surface. Due to the absence of the interface meshes, contact angle cannot be measure and/or prescribed in a straight-forward way. 
Some of the above-described issues may be efficiently resolved by applying kinematic frameworks that are relying on mesh-based description of the moving interface between the phases to the problem at hand.

\subsection{Moving grid models}

Fully moving grid models An alternative to fixed-grid (Eulerian) models are the moving-grid ones, such as Lagrangian or Arbitrary Lagrangian/Eulerian (ALE). Upto-date these models have not been applied to the simulation of droplet-airflow interaction in fuel cell channels. This may be attributed to the fact that moving-grid models are practically absent in almost all the most used commercial (ANSYS Fluent [2], ANSYS CFX 1], COMSOL Multiphysics [3]) and open source (OpenFOAM [5], OpenFVM [6], Elmer [4]) CFD software. Nevertheless, moving grid models have been successfully applied to general multi-phase and droplet dynamics problems.

The idea of the moving grid methods for liquid dropletair interaction problems is to discretize the entire heterogeneous domain by a unique grid, where the subdomains corresponding to the liquid and the gas share the mesh at the interface. The evolution of the interface location is directly defined by the motion of the corresponding grid. Thus, no additional techniques for detecting the interface are necessary. A schematic of a moving-grid model is shown in Fig. 13 . The momentum equation of a fluid written with respect to the moving grid reads 36:

$\rho \frac{\partial \mathbf{v}}{\partial t}+\rho(\mathbf{c} \cdot \nabla) \mathbf{v}-\mu \nabla \cdot\left(\nabla \mathbf{v}+\nabla^{T}(\mathbf{v})\right)+\nabla p=\mathbf{f}_{e x t}$

The convection term now contains the velocity $\mathbf{c}=$ $\mathbf{v}-\mathbf{v}_{m}$, where $\mathbf{v}_{m}$ is the mesh velocity. In case of zero mesh velocity one recovers the standard fixed grid (Eulerian) equations, while in case of mesh and convection velocities being equal (material "attached" to the mesh) one obtains a fully Lagrangian case.

ALE models for two-phase flow analysis can be found in $82,83,122$. In 82 an efficient reconnection algorithm is proposed for the interface. ALE models reach their limit when the mesh deformations become large and the element degradation or even inversion takes place. In such cases re-meshing must be performed.

As a particular class of ALE methods, purely Lagrangian two-phase approaches have been proposed in 57 . 69,88 . In these methods re-meshing is included as an obligatory ingredient of the overall strategy. Theoretically, such approaches allow treating arbitrary mesh deformations. However, impossibility of mesh quality control in fully Lagrangian methods as well as necessity of re-meshing the entire computational domain (air and liquid) practically lead to large computational cost, particularly in 3D.

Moreover, ALE and Lagrangian approaches rely on boundary-conforming meshes, meaning that the interface nodes are shared by the air and liquid sub-domains. This introduces a severe difficulty for representing the discontinuity of the flow variables across the interface between the phases. In case of strong discontinuity in pressure due to non-negligible surface tension, one must duplicate the degrees of freedom at the interface 69,88 .

In literature several studies analyzed droplets excluding the air domain by Lagrangian models, such as the one proposed by Saksono and Peric [111. Saksono and Perić [111] proposed an implicit variational formulation for the surface tension term. This formulation could be used to describe quasistatic and dynamic problems [112]. However, the model relied upon an axisymmetric formulation, not being able to solve complex geometries.

Pure moving grid methods have not been employed to the problem at hand to-date. Nevetheless, it is important to point out that their potential is obvious. Particularly, employing an ALE methodology using a single mesh for the two phases, where the droplet and the nodes in the vicinity to the air-liquid interface are treated in a nearly Lagrangian fashion, while the external boundaries (channel walls and GDL surface) are treated in an Eulerian framework defines a promising strategy.

Models combining fixed and moving grids Embedded models rely on combining an Eulerian and a Lagrangian description for gas and liquid, respectively. The overall problem becomes split and is solved in a partitioned manner, which offers a considerable improvement in computational efficiency in comparison with the monolithic VOF methods, where liquid and gas are solved in a single system. This approach has been recently introduced in the community of CFD developers and has shown numerous advantages when applied to modeling of two-phase transport in the fuel cells. In the next section, the model is described in detail and its results are summarized.

\section{Embedded model for two-phase flows}

A novel numerical approach relying on combining fixedgrid and moving-grid models for the gas and the liquid, respectively, was proposed by Ryzhakov et al in 108. In the original paper the method was developed for a general liquid-gas system and proved to be particularly advantageous for problems with strong discontinuities across the phase interface, such as those caused 


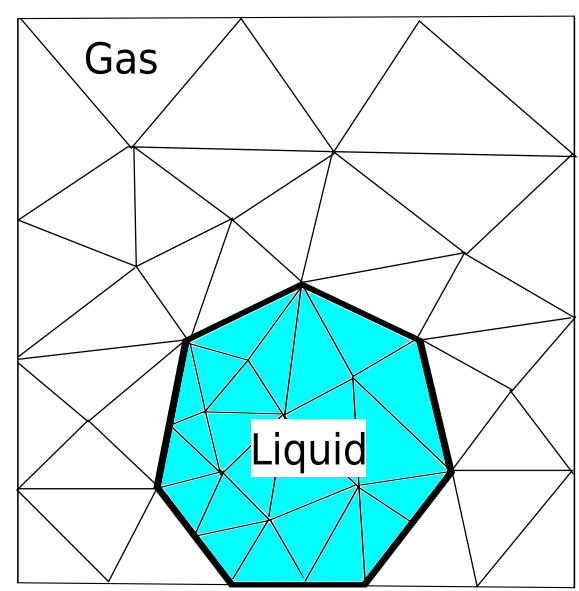

(a) Initial domain configuration

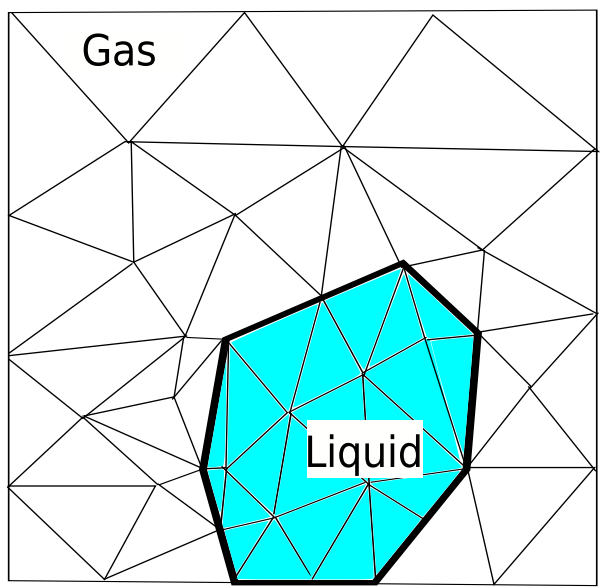

(b) Deformed domain configuration

Fig. 13 A schematic of a moving grid approach for a droplet-air system

by surface tension effects. The idea consisted in embedding a Lagrangian model describing the liquid into an Eulerian model for the surrounding gas (airflow). The Lagrangian domain (liquid) moved on top of the fixed Eulerian mesh forcing the surrounding fluid to move accordingly. The location of the material interface was accurately defined by the intersection of the boundary mesh of the Lagrangian domain with the Eulerian grid. Gas and liquid solvers were coupled using a Dirichlet-Neumann algorithm. Representation of the pressure and/or pressure gradient discontinuity across the interface did not require any additional techniques being an intrinsic feature of the method. Below the governing equations of the embedded model as well as the solution algorithm are presented.

\subsection{Governing equations}

The embedded setting for a liquid-gas system consists of two overlapping domains: the one with the fixed boundaries (gas) and the one with the moving boundaries (liquid). Let us describe this setting at the continuum level first. Let us consider Lagrangian domain $\Omega_{L}$ (representing liquid) embedded into the Eulerian domain $\Omega_{E}$ with an external boundary $\Gamma_{E}$ (see Fig. 14). Note that $\Omega_{E}$ is the entire rectangular domain, including the subdomain overlapping with Lagrangian domain and the subdomain lying outside and representing the actual gas. In the embedded setting the interface $\Gamma_{I}$ between the two fluids is defined by the position of the boundary of the Lagrangian domain $\Gamma_{L}$. The interface $\Gamma_{I}$ splits the Eulerian domain into two parts: the real one $\Omega_{E}^{r}$ (representing the gas and shown in white in the figure)

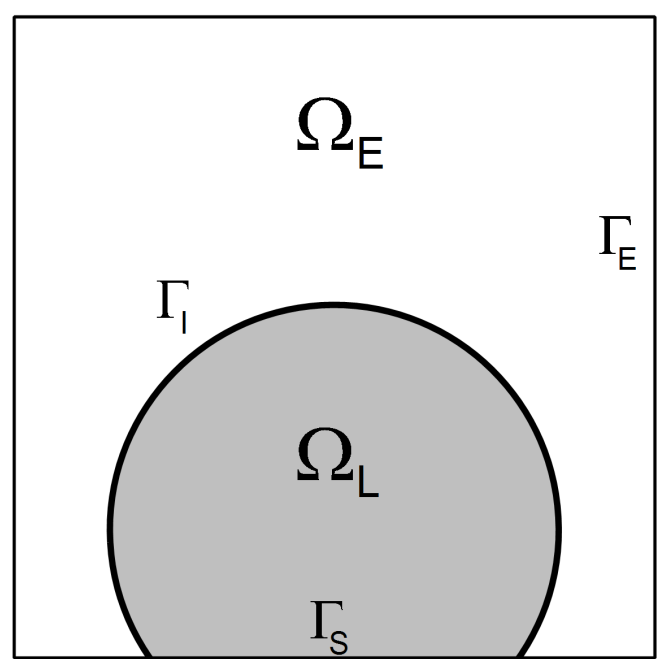

Fig. 14 Gas and liquid domains at continuum level

and the fictitious one $\Omega_{E}^{f}$ that does not have physical meaning. At continuous level the fictitious Eulerian domain exactly coincides with the Lagrangian domain and $\Gamma_{I}=\Gamma_{L} . \Gamma_{s}$ defines the boundary of the liquid phase that in contact with the solid.

The governing system in either domain is the NavierStokes equations equipped with the incompressibility condition (Eqs. 100, 11).

The physical properties are defined as $\rho=\rho_{l}$ in $\Omega_{L}$ and $\rho=\rho_{g}$ otherwise (indices "l" and "g" stand for liquid and gas, respectively). 


\subsubsection{Boundary and interface conditions}

In order to ensure the well-posedness of the NavierStokes problem defined by Eqs. (10), (11) suitable boundary conditions must be specified. On the external boundary $\Gamma_{E}=\Gamma_{D} \cup \Gamma_{N}$, such that $\Gamma_{D} \cap \Gamma_{N}=\emptyset$, the following conditions are prescribed:

$$
\begin{array}{ll}
\mathbf{v}=\mathbf{v}^{p r} & \text { at } \Gamma_{D} \\
\boldsymbol{\sigma} \cdot \mathbf{n}=\boldsymbol{\sigma}_{n}^{p r} & \text { at } \Gamma_{N}
\end{array}
$$

where $\mathbf{v}^{p r}$ is the prescribed velocity, $\boldsymbol{\sigma}$ is the Cauchy stress tensor, and $\boldsymbol{\sigma}_{n}^{p r}$ is the prescribed traction vector. For an incompressible Newtonian fluid, $\boldsymbol{\sigma}$ reads:

$$
\boldsymbol{\sigma}=-p \boldsymbol{I}+\mu\left(\nabla \mathbf{v}+\nabla^{T} \mathbf{v}\right)
$$

where $\boldsymbol{I}$ is the unit tensor. On the internal interface $\Gamma_{I}$ the coupling conditions are:

$$
\begin{array}{ll}
\llbracket \mathbf{v} \rrbracket=0 & \text { at } \Gamma_{I} \\
\llbracket \boldsymbol{\sigma} \rrbracket \cdot \mathbf{n}=\gamma \kappa \mathbf{n} & \text { at } \Gamma_{I}
\end{array}
$$

where $\mathbf{n}$ now is the unit normal to the interface $\Gamma_{I}$, $\gamma$ and $\kappa$ are the surface tension coefficient and the interface curvature, respectively. The $\llbracket x \rrbracket$ symbol represents the jump in the quantity $x$ across the interface.

Eq. (24) expresses the continuity of all velocity components $\left(\llbracket \mathbf{v} \rrbracket=\mathbf{v}_{E}-\mathbf{v}_{L}\right.$, where indices $E$ and $L$ distinguish the quantities corresponding to the Eulerian fluid (gas) and the Lagrangian fluid (liquid), respectively). The equality of the normal components of velocity ensures no mass flow across the interface. The tangential components' equality is similar to a no-slip condition and is necessary when fluids with non-zero viscosity are considered. Eq. 25 expresses that the difference in the normal stress across the interface is balanced by the surface tension force.

Projecting Eq. 25 onto the normal and tangential directions leads to the following scalar interface conditions:

$$
\begin{array}{ll}
\mathbf{n} \cdot \llbracket \boldsymbol{\sigma} \rrbracket \cdot \mathbf{n}=\gamma \kappa & \text { at } \Gamma_{I} \\
\mathbf{t} \cdot \llbracket \boldsymbol{\sigma} \rrbracket \cdot \mathbf{n}=0 & \text { at } \Gamma_{I}
\end{array}
$$

Noting that the jump in the stress across the interface is equal to the difference between the stresses of the two fluids $\llbracket \boldsymbol{\sigma} \rrbracket=\boldsymbol{\sigma}_{E}-\boldsymbol{\sigma}_{L}$ and splitting the stress tensor into volumetric and deviatoric part using Eq. 23 results in:

$$
\begin{aligned}
& \left(p_{E}-p_{L}\right)+\mu_{E} \mathbf{n} \cdot\left[\nabla \mathbf{v}+\nabla^{T} \mathbf{v}\right]_{E} \cdot \mathbf{n}- \\
& \mu_{L} \cdot \mathbf{n} \cdot\left[\nabla \mathbf{v}+\nabla^{T} \mathbf{v}\right]_{L} \cdot \mathbf{n}=-\gamma \kappa \\
& \mu_{E} \mathbf{t} \cdot\left[\nabla \mathbf{v}+\nabla^{T} \mathbf{v}\right]_{E} \cdot \mathbf{n}- \\
& \mu_{L} \cdot \mathbf{t} \cdot\left[\nabla \mathbf{v}+\nabla^{T} \mathbf{v}\right]_{L} \cdot \mathbf{n}=0
\end{aligned}
$$

\subsection{Discretization}

According to the embedded setting for two-phase flows, liquid is discretized by a moving (Lagrangian) grid, while equations for the gas are solved on a fixed Eulerian mesh. Eulerian grid, however, covers the entire computational domain. This setting and resulting partitioning is indicated in Fig. 15 .

As shown in Fig. 15 Eulerian mesh is split into two parts by the surface mesh of the liquid domain. The first one, $\Omega_{E}$, corresponds represents actual gas. The second one, $\Omega_{E}^{f}$, is considered fictitious on the Eulerian mesh as the actual liquid occupying this sub-domain is modeled on the Lagrangian mesh. In order to find the partitioning of the Eulerian domain, the intersections of the Lagrangian surface mesh $\Gamma_{L}$ with the Eulerian mesh is identified. The polyline/polygon defined by these intersections provides $\Gamma_{I}$, i.e., the representation of the Lagrangian surface within the Eulerian mesh.

The interaction of the two domains is represented by interchanging boundary conditions at the interface. Moving liquid provides the Dirichlet condition for the gas (velocity of the surface of the liquid), while stresses in the gas phase provide the Neumann condition for the liquid. In what follows, discrete version of governing equations for both domains are presented and the coupling strategy is specified.

\subsubsection{Eulerian model (gas)}

The "real" elements of the Eulerian mesh are governed by the Navier-Stokes equations. The discrete version of these equations (obtained using linear velocity-pressure Finite Elements in space and Backward Euler scheme in time) in the residual form reads (see 108 for the derivation):

$$
\begin{aligned}
& \overline{\mathbf{r}}_{E}^{m}=0 \\
& \overline{\mathbf{r}}_{E}^{c}=0
\end{aligned}
$$

where $\overline{\mathbf{r}}^{m}$ and $\overline{\mathbf{r}}^{c}$ are the residua of the momentum and continuity equations, respectively. Note that the subindex $E$ stands for Eulerian. The residua are defined as:

$$
\begin{aligned}
\overline{\mathbf{r}}_{E}^{m}= & \mathbf{F}_{n+1}-\rho \mathbf{M} \frac{\overline{\mathbf{v}}_{n+1}-\overline{\mathbf{v}}_{n}}{\Delta t}-\overline{\mathbf{K}}\left(\overline{\mathbf{v}}_{n+1}\right) \overline{\mathbf{v}}_{n+1}+ \\
& \mu \mathbf{L} \overline{\mathbf{v}}_{n+1}-\mathbf{G} \overline{\mathbf{p}}_{n+1} \\
\overline{\mathbf{r}}_{E}^{c}= & \mathbf{D} \overline{\mathbf{v}}_{n+1}+\mathbf{S} \overline{\mathbf{p}}_{n+1}
\end{aligned}
$$

where $\mathbf{M}, \mathbf{L}, \mathbf{G}$ and $\mathbf{D}$ are mass, Laplacian, gradient and divergence matrices, respectively. $\overline{\mathbf{v}}$ and $\overline{\mathbf{p}}$ are the 


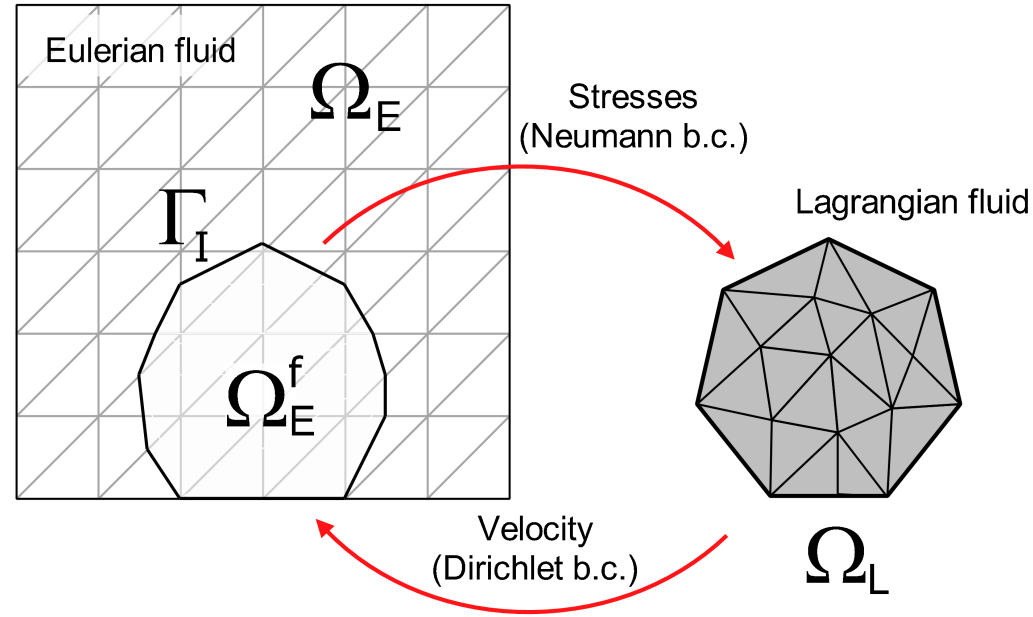

Fig. 15 Embedded partitioning: Lagrangian domain (liquid) immersed into the Eulerian domain

nodal velocity and the nodal pressure vectors, respectively and $\overline{\mathbf{F}}$ is the body force vector.

The matrices and vectors are assembled from the elemental contributions defined as

$$
\begin{aligned}
\mathbf{M} & =\sum_{\text {elem }} \int_{\Omega_{e}} \mathbf{N N}^{T} d \Omega \\
\mathbf{L} & =\sum_{\text {elem }} \int_{\Omega_{e}} \nabla \mathbf{N} \nabla \mathbf{N}^{T} d \Omega \\
\mathbf{G} & =-\sum_{\text {elem }} \int_{\Omega_{e}} \nabla \mathbf{N N}_{p} d \Omega \\
\overline{\mathbf{F}}= & \sum_{\text {elem }} \rho \int_{\Omega_{e}} \mathbf{N g} d \Omega \\
\mathbf{S}= & \sum_{\text {elem }} \int_{\Omega_{e}} \tau \nabla \mathbf{N}{ }_{p} \nabla \mathbf{N}_{p}^{T} d \Omega \\
\mathbf{D}= & -\mathbf{G}^{T} \\
\mathbf{K}= & \sum_{\text {elem }}\left(\rho \int_{\Omega_{e}} \mathbf{N}(\overline{\mathbf{v}} \cdot \nabla \mathbf{N}) d \Omega+\right. \\
& \left.\int_{\Omega_{e}}(\overline{\mathbf{v}} \cdot \nabla \mathbf{N}) \tau\left(\frac{\rho}{\Delta t} \mathbf{N}+\rho \overline{\mathbf{v}} \cdot \nabla \mathbf{N}\right) d \Omega\right)
\end{aligned}
$$

where $\mathbf{N}$ and $\mathbf{N}_{p}$ stand for linear FE shape functions for the velocity and the pressure variables, respectively. The second term in the definition of $\mathbf{K}$ corresponds to the convection stabilization according to SUPG (Streamline Upwind Petrov-Galerkin) method [119]. Matrix $\mathbf{S}$ is the pressure stabilization term necessary due to using equal order interpolations for the velocity and the pressure [54]. Algorithmic stabilization parameter is computed as $\tau=\left(\frac{h}{2\|\mathbf{v}\|}+\frac{h^{2}}{4 \nu}\right)^{-1}$, where $h$ is the element size, and $\nu$ is the kinematic viscosity of the fluid.

Discretized governing system defined Eqs. 30, 31 is non-linear due to the dependence of the convection operator on the unknown velocity. Therefore, discretized equations are linearized using the Newton-Raphson method. The linearized system solved at every step of the iterative procedure can be written as:

$\mathbf{H}_{E}\left(\begin{array}{l}\delta \overline{\mathbf{v}} \\ \delta \overline{\mathbf{p}}\end{array}\right)=\left(\begin{array}{c}\overline{\mathbf{r}}_{m}^{f e} \\ \overline{\mathbf{r}}_{c}^{f e}\end{array}\right)$

where the tangent matrix $\mathbf{H}_{E}$ is defined as:

$\mathbf{H}_{E}=\left(\begin{array}{cc}\frac{\mathbf{M}}{\Delta t}+\rho \overline{\mathbf{K}}+\mathbf{S}_{K}+\mu \mathbf{L} & \mathbf{G} \\ \mathbf{D} & \tau \mathbf{L}\end{array}\right)$

The above equations must be equipped with a boundary condition representing the velocity of the Lagrangian domain (liquid) in the Eulerian mesh, i.e., $\mathbf{v}_{E}-\mathbf{v}_{L}=0$ at $\Gamma_{I}$. The approximation of this condition is specified in Section 4.3 .

Once $\delta \overline{\mathbf{v}}$ and $\delta \overline{\mathbf{p}}$ are found, the primary variables are updated as $\overline{\mathbf{v}}_{n+1}^{i+1}=\overline{\mathbf{v}}_{n+1}^{i}+\delta \overline{\mathbf{v}}$ and $\overline{\mathbf{p}}_{n+1}^{i+1}=\overline{\mathbf{p}}_{n+1}^{i}+\delta \overline{\mathbf{p}}$ (where $i$ is the non-linear iteration index) until convergence is achieved. Instead of solving the governing system monolithically, fractional step method 26] decoupling velocity and pressure can be used in order to reduce the computational cost. Implementation of the fractional step method in the present context is shown in 61, 108]. For using in the contexts of a different class of time integration schemes fractional step method can be consulted in 107.

\subsubsection{Lagrangian model (liquid)}

A boundary conforming Lagrangian formulation is used for modeling the liquid phase according to the embed- 
ded method for two-phase flows. The discrete version of the residual form of Navier-Stokes in the updated Lagrangian framework reads:

$\overline{\mathbf{r}}_{L}^{m}=\overline{\mathbf{F}}+\overline{\mathbf{F}}_{i n t}-\left(\mathbf{M} \frac{\overline{\mathbf{v}}_{n+1}-\overline{\mathbf{v}}_{n}}{\Delta t}+\mu \mathbf{L} \overline{\mathbf{v}}_{n+1}+\mathbf{G} \overline{\mathbf{p}}_{n+1}\right)$

$\overline{\mathbf{r}}_{L}^{c}=\mathbf{D} \overline{\mathbf{v}}_{n+1}+\mathbf{S}_{D} \overline{\mathbf{p}}_{n+1}$

According to the updated Lagrangain framework the discrete operators (defined by Eqs. (34)-(40)), are now calculated using the deformed configuration $\mathbf{X}_{n+1}$.

The force $\overline{\mathbf{F}}_{\text {int }}$ in Eq. (43) is the interface Neumann term representing the normal and shear stress components exerted by the gas upon the liquid and the surface tension. It corresponds the interface condition defined by Eqs. 26) and (27). $\overline{\mathbf{F}}_{\text {int }}$ is computed as

$\overline{\mathbf{F}}_{\text {int }}=\overline{\mathbf{F}}_{N}+\overline{\mathbf{F}}_{s t}+\overline{\mathbf{F}}_{s h} \quad$ on $\Gamma_{L}$

where

$$
\begin{aligned}
\overline{\mathbf{F}}_{N}= & -\int_{\Gamma_{L}} \mathbf{N} p_{E} \cdot \mathbf{n} \mathrm{d} \Gamma_{L}+ \\
& \mu_{E} \int_{\Gamma_{L}}\left(\nabla(\mathbf{N} \overline{\mathbf{v}})+\nabla(\mathbf{N} \overline{\mathbf{v}})^{T}\right)_{E} \cdot \mathbf{n} \mathrm{d} \Gamma_{L} \\
\overline{\mathbf{F}}_{s t}= & -\int_{\partial \Gamma} \gamma \mathbf{m} \cdot \mathbf{N} \mathrm{d}(\partial \Gamma)-\int_{\Gamma_{L}} \mathbf{N} \gamma \kappa \cdot \mathbf{n} \mathrm{d} \Gamma_{L} \\
\overline{\mathbf{F}}_{s h}= & \mu_{E} \int_{\Gamma_{L}}\left(\nabla(\mathbf{N} \overline{\mathbf{v}})+\nabla(\mathbf{N} \overline{\mathbf{v}})^{T}\right)_{E} \cdot \mathbf{m} \mathrm{d} \Gamma_{L}
\end{aligned}
$$

where $\mathbf{m}$ is the vector tangent to $\Gamma$, i.e., perpendicular to $\mathbf{n}$.

The integrands in the terms $\mathbf{F}_{N}$ and $\mathbf{F}_{s h}$ are computed in the interface elements of the Eulerian mesh and then are projected onto $\Gamma_{L}$, where the integrals are computed. The surface tension force is computed directly on the Lagrangian surface mesh.

The surface tension term along the contact line (in $3 \mathrm{D})$ or triple points (2D) requires special treatment, since straight-forward calculation of the curvature in this location would result in a zero value. Thus, the curvature at these nodes is computed using the normal vector at the contact line $\left(\mathbf{n}_{1}\right.$ in Fig. 16(c) $)$ and the normal vector at its nearest neighbor node from $\Gamma_{L}$ (node 2 in Fig. 16(c) as:

$\kappa=\left\|\frac{\mathrm{d} \mathbf{n}}{\mathrm{d} s}\right\|=\frac{\left\|\mathbf{n}_{1}-\mathbf{n}_{2}\right\|}{\mathrm{d} s}$

where $\mathrm{d} s$ is the distance between nodes 1 and 2 .

When a droplet lays on a rough surface, such as those used in PEFC gas channels, the concept of static
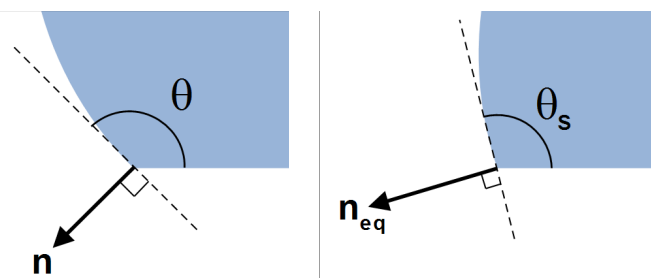

(a) Current configuration (b) Equilibrium configuration

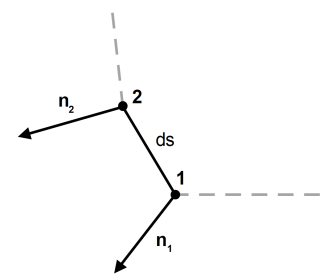

(c) Normal vector at contact line node and its nearest neighbor

Fig. 16 (a)-(b) Normal vector and contact angle at current and equilibrium configurations and (c) Normal vector at contact line node and its nearest neighbor to obtain the curvature at contact line

contact angle cannot be used. In rough surfaces, the contact line pins and the contact angle changes from one equilibrium configuration to another. According to 61] two threshold values must be used: $\theta_{\min }$ and $\theta_{\max }$, as contact angle conditions. The contact line is fixed only within the range $\theta \in\left[\theta_{\min }, \theta_{\max }\right]$. These maximum and minimum values represent the experimentally measured contact angles for incipient motion when the droplet is placed on a tilted plane of a given material.

When the dynamic contact angle condition is not fulfilled (i.e., $\theta<\theta_{\min }$ or $\theta>\theta_{\max }$ ), the contact line is allowed to move. Instead of the no-slip boundary condition, a slip boundary condition is applied at those nodes representing the contact line:

$\mathbf{v} \cdot \mathbf{n}=0$

Even though incompressible Navier-Stokes equations are often solved by pressure segregation (fractional step) methods, it has been shown by Ryzhakov et al in 108 that for the problems where surface tension is not negligible, typical version of such techniques lead to spurious oscillations of the boundary. Thus, unlike the governing system for the gas phase, the the governing equations for the liquid must be solved in a coupled (monolithic) fashion.

Eqs. (43), 44) are non-linear since in the updated Lagrangian setting the discrete operators (Eqs. (34)(40) ) correspond to the unknown current configuration 
$\left(\mathbf{X}_{n+1}\right)$ according to the updated Lagrangian framework. Therefore, the governing equations' system must be solved iteratively, updating the operators at every iteration $i$.

The linearized system can be written as

$$
\mathbf{H}_{L}\left(\begin{array}{c}
\delta \overline{\mathbf{v}} \\
\delta \overline{\mathbf{p}}
\end{array}\right)=\left(\begin{array}{c}
\overline{\mathbf{r}}_{L}^{m} \\
\overline{\mathbf{r}}_{L}^{c}
\end{array}\right)
$$

where the tangent matrix $\mathbf{H}_{L}$ is defined as:

$$
\mathbf{H}_{L}=\left(\begin{array}{cc}
\frac{\mathbf{M}}{\Delta t}+\mu \mathbf{L}+\mathbf{H}_{s t} & \mathbf{G} \\
\mathbf{D} & \tau \mathbf{L}
\end{array}\right)
$$

The term $\mathbf{H}_{s t}$ in Eq. (52) has the following expression:

$$
\begin{aligned}
\mathbf{H}_{S T}= & -\gamma \Delta t\left[\int_{\partial \Gamma}(\mathbf{N} \delta \overline{\mathbf{v}}) \mathbf{N} \mathrm{d}(\partial \Gamma)+\right. \\
& \left.\int_{\Gamma} \nabla_{s}(\mathbf{N} \delta \overline{\mathbf{v}}) \nabla_{s} \mathbf{N} \mathrm{d} \Gamma\right]
\end{aligned}
$$

where $\nabla_{s}$ is the tangential gradient operator. For a scalar function $f$, this operator is defined as follows:

$\nabla_{s} f(x)=\nabla f(x)-\mathbf{n} \otimes \mathbf{n} \cdot \nabla f(x)$

The term $\mathbf{H}_{s t}$ is the result of linearization of surface tension term $\mathbf{F}_{s t}$ defined in Eq. (47). Considering $\mathbf{H}_{s t}=$ 0 , an explicit version of integration of surface tension is obtained. For the problem at hand, implicit surface tension treatment is of essential importance as it allows to circumvent the sever time step size restrictions faced by explicit formulations. For the derivation of $\mathbf{H}_{s t}$ and the $3 \mathrm{D}$ version of the corresponding term the reader is referred to Chapter 3 of [59] or [60].

Once the system (Eq. (51) is solved, the velocity and the pressure are updated as $\overline{\mathbf{v}}_{n+1}^{i+1}=\overline{\mathbf{v}}_{n+1}^{i}+\delta \overline{\mathbf{v}}$ and $\overline{\mathbf{p}}_{n+1}^{i+1}=\overline{\mathbf{p}}_{n+1}^{i}+\delta \overline{\mathbf{p}}$. The position of the nodes in $\Omega_{L}$ is updated as $\mathbf{X}_{n+1}^{i+1}=\mathbf{X}_{n}+\Delta t \cdot \overline{\mathbf{v}}_{n+1}^{i+1}$.

The integration domains necessary for the computation of the discrete operators (Eqs. (34)- 40) are be updated according to this new configuration $\mathbf{X}_{n+1}^{i+1}$.

\subsection{Coupling and overall solution algorithm}

Lets us consider the Eulerian and the Lagrangian domains discretized with a finite element mesh. Fig. 17(a) shows the overlapping meshes. One can distinguish the boundary of the Lagrangian domain $\Gamma_{L}$ and its representation on the Eulerian mesh $\Gamma_{I}$. Note that as the element size $h \rightarrow 0$ in both domains, the two boundaries tend to coincide: $\Gamma_{I} \rightarrow \Gamma_{L}$. The embedded interface $\Gamma_{I}$ (see black polyline in Fig. 17(b) splits the Eulerian domain into real and fictitious parts $\Omega_{E}^{r}$ and $\Omega_{E}^{f}$, as already mentioned. We shall denominate the nodes and the elements contained in $\Omega_{E}^{r}$ and $\Omega_{E}^{f}$ "real" and "fictitious", respectively (see Fig. 17(b)]. We also distinguish the "interface elements", i.e., the elements cut by $\Gamma_{I}$ and thus containing both the real and the fictitious nodes. This is shown in Fig. 17(c) where the interface elements are shown in gray, fictitious and real nodes are indicated by black and gray dots, respectively.

The interface $\Gamma_{I}$ intersects the Eulerian mesh at arbitrary positions, not necessarily at the nodes (see Fig. 17). Without introducing the element splitting, the interface Dirichlet boundary condition can be applied in a "weak" sense, i.e. minimizing the difference between the velocity of the liquid and the gas along the interface $\Gamma_{I}$. The interface Dirichlet boundary condition can thus be applied at the existing fictitious nodes of the interface elements ${ }^{3}$ (see black dots in the interface elements in Fig. 17(c) . This way, only the existing degrees of freedom are used and thus the global matrix structure corresponding the Eulerian domain remains unaltered 27, 86 .

Given the velocity $\mathbf{v}_{L}$ of the liquid at its boundary, the difference to be minimized is given by (see e.g. 27 or [106] for details)

$\int_{\Gamma_{I}} \omega\left(\mathbf{v}_{E}-\mathbf{v}_{L}\right) d \Gamma_{I}=0$

where $\mathbf{v}_{E}$ and $\mathbf{v}_{L}$ are the velocities of the Eulerian and Lagrangian domains at the interface, respectively, and $\omega=\mathbf{N}^{T} q$ is the velocity test function.

After velocity discretization $(\mathbf{v}=\mathbf{N} \overline{\mathbf{v}})$ Eq. (55) leads to:

$\mathbf{M}_{\Gamma_{I}} \overline{\mathbf{v}}_{n+1}=\mathbf{f}_{\Gamma_{I}}$

where $\overline{\mathbf{v}}_{n+1}$ is the velocity of the nodes of the interface elements, $\mathbf{M}_{\Gamma_{I}}=\int_{\Gamma_{I}} \mathbf{N}^{T} \mathbf{N} d \Gamma_{I}$ and $\mathbf{f}_{\Gamma_{I}}=\int_{\Gamma_{I}} \mathbf{N}_{f}^{T} \mathbf{v}_{L} d \Gamma_{I}$, where $N_{f}$ are the shape functions corresponding to the fictitious nodes.

Eq. (56) provides a constraint to the governing system for the gas phase (Eq. 41)) representing the effect of the velocity of the embedded Lagrangian domain in the Eulerian mesh.

Solving Eq. (56) the velocity at the fictitious nodes is obtained, that is consequently applied as the interface Dirichlet boundary condition at the next time step, representing the action of the Lagrangian fluid onto the Eulerian one. Neumann part of the coupling, representing the stresses exerted by the gas onto the liquid phase, has been previously described (Eq. 46, 48).

3 This can be understood as follows: apply a velocity at fictitious nodes of the Eulerian interface elements that minimizes the difference between the velocity of the Eulerian fluid (gas) and the liquid along the interface in the least square sense. 


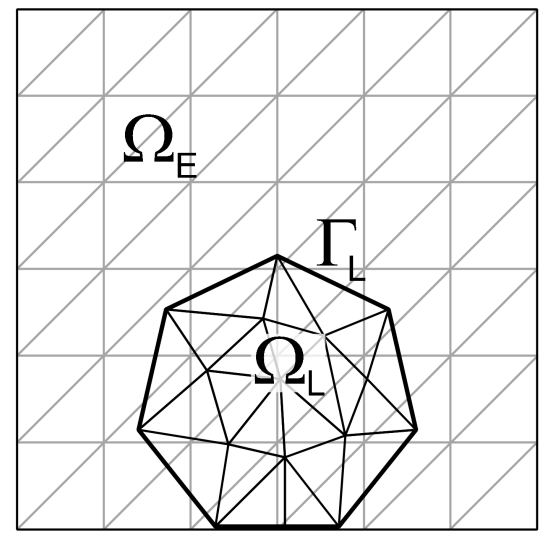

(a) Superimposed discretized domains

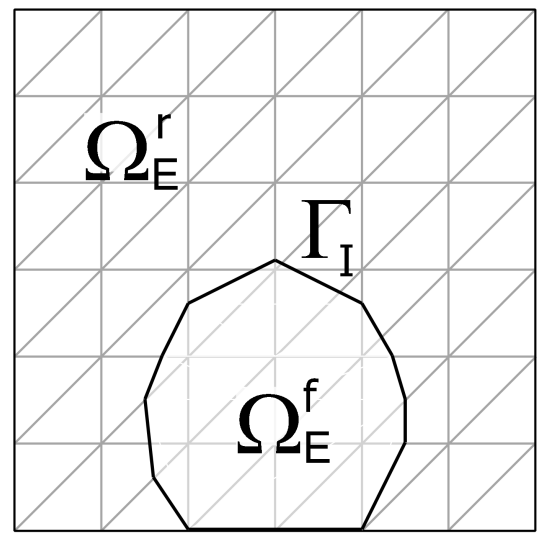

(b) Interface on the Eulerian mesh

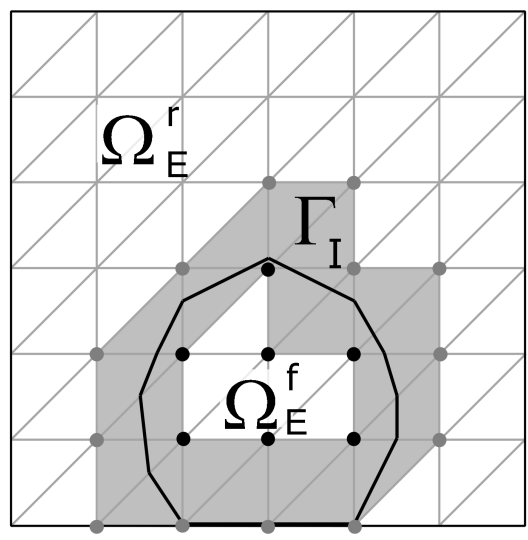

(c) Interface elements

Fig. 17 Embedded setting: real, fictitious and interface parts of the Eulerian domain

Considering that at the time step $t_{n}$ the solution (velocity $\overline{\mathbf{v}}_{n}$ and pressure $\overline{\mathbf{p}}_{n}$ ) is known in both the domains $\Omega_{E}$ and $\Omega_{L}$, one obtains the velocity and pressure fields at $t_{n+1}$ according to the strategy presented in Table 1 .

Table 1 Implementation procedure of an embedded approach applied to the solution of liquid-gas interaction

1. Solve the liquid problem on the Lagrangian mesh (Eq. (51) ) and update the Lagrangian mesh position.

Output: new position of the Lagrangian mesh, $\overline{\mathbf{v}}_{n+1}$ $\overline{\mathbf{p}}_{n+1}$ in $\Omega_{L}$

2. Identify the position of Lagrangian domain within the Eulerian one. Identify the interface elements, the "real" and the "fictitious" elements and nodes. Output: $\Gamma_{I}$

3. "Switch off" the fictitious Eulerian elements $\left(\Omega_{E}^{f}\right)$.

4. Solve the gas problem on the Eulerian mesh (Eq. (41)) equipped with the interface Dirichlet boundary representing velocity of the boundary of the liquid phase

Output: velocity and pressure $\overline{\mathbf{v}}_{n+1}$ and $\overline{\mathbf{p}}_{n+1}$ in $\Omega_{E}$.

5. Project the air stresses onto the liquid boundary $\Gamma_{L}$ surface and compute the corresponding force term $\overline{\mathbf{F}}_{\text {int }}$ (Eq. 45 ) for the momentum equation of the liquid 43).

6. Go to next time step

\subsection{Numerical simulations results and discussion}

Numerical validation of the embedded model in application to a general gas-liquid problem can be found in [108]. The static droplet benchmark was solved using the embedded method and the fully Lagrangian formulation from reference 57. The former proved to obtain stable solution in the vicinity of the material interface, while pressure instability and spurious velocities were observed in the latter. Two dynamic problems were also studied and the embedded formulation could a) capture the frequency of oscillation of a free droplet and b) obtain good agreement with numerical results in literature of the predicted air pressure profile under a levitating droplet.

The model was experimentally validated for sessile droplets in 61]. Several droplets with varying volumes were injected on a rough surface and numerical results had excellent agreement with experimental observations. The deformation of a sessile droplet on an inclined plane was also predicted with good agreement. An additional example was included to compare droplet oscillation with experimental data from literature and study effects of substrate nature on droplet deformation and detachment.

The proposed technique for representing the wetting characteristics of the solid via contact angle condition was tested for different substrates in 109, giving results for equilibrium contact angle with less than $1 \%$ error from the prescribed value. The contact angle was not prescribed, but it was rather included in the surface tension force term computed in the contact line. A technique for modeling liquid injection through a pore based on "Lagrangian nodal inlet" technique was introduced, and the model was used to predict droplet growth and deformation. Results were compared to experimental data from literature and results were in reasonable agreement, specially at early stages of injection, i.e., prior to the onset of the separation (detachment) of the liquid phase from the substrate. 
Rigorous derivation of the associated implicit surface tension model and corresponding validation was presented in 60 . In this paper it was shown that the proposed surface tension model alleviates the severe time step restrictions encountered in explicit surface tension models, such as the ones used practically in all the VOF simulations described in Section 3.1. It was shown that stable solution could be obtained for time step sizes of up to 2 orders of magnitude higher, that those of the explicit models applied to the same problem and same spatial discretization. It was also concluded that in case of using the implicit treatment of surface tension, the time step ensuring convergence becomes governed by the non-linearity of the problem and not by the time-scale of the capillary waves.

Next, the details of several numerical studies using the embedded model are presented emphasizing the new insights obtained. Properties of the fluids used in these examples are displayed in Table 2. These values correspond to a constant temperature of $298 \mathrm{~K}$ and a pressure of $1 \mathrm{~atm}$.

\subsubsection{Effect of droplet volume on frequency of oscillations}

Recent studies on droplet dynamics in PEFC show that oscillations are responsible for droplet detachment 39 , 62, 89. The frequency of oscillation of a sessile droplet subjected to an airflow is studied in this example. Numerical results are then compared to experimental data reported by Milne 89.

The numerical setup is a rectangular channel with a sessile droplet on a Teflon surface placed in the center. Oscillations for different droplet volumes, i.e., 13, 30, 58 and $100 \mu \mathrm{l}$, are measured with an airflow increasing velocity. For each case, the droplet is considered to have a constant volume. A Dirichlet boundary condition for airflow velocity is applied at the channel entry. An open-boundary (no shear stress) boundary condition is applied to the channel outlet, and no-slip boundary conditions are applied to the rest of channel walls. Channel size as well as flow parameters are detailed in Table 3. Simulations have been performed with a time step $\delta t=10^{-3} \mathrm{~s}$, and the simulation time is $1 \mathrm{~s}$. Further details on the computational domain, as well as mesh sizes, can be found in reference 61.

Pressure and velocity distributions at steady-state for a $13 \mu$ l-volume droplet are depicted in Figs. 18(a) and $18(\mathrm{~b})$, respectively. Pressure exhibits a nearly hydrostatic profile within the droplet, with mild oscillations due to the drag exerted by air. A recirculation pattern for velocity distribution can be observed in Fig. 18(b), This phenomenon has been observed experimentally in reference 90 .

Airflow velocity distribution at the wake of the droplet shows vortex formation. At steady state, pressure is higher on the upstream side of the droplet, and low pressure and velocity values are observed on the wake side. A recirculation pattern for velocity is observed in this region, as depicted in Fig. 18(c). Viscous stress and pressure difference across the droplet deform the droplet in the downstream direction. Surface tension force act as a restoring force, acting on the opposite direction of external forces. Consequently, the droplet oscillates due to this unbalancing and balancing process.

Fig. 18(d) shows the frequency of oscillation in $x$ and $y$ directions observed at the droplet's center of mass for several droplet volumes. Numerical results (square and triangle markers) are compared to experimental data (represented by solid and dashed lines) from reference 89. No significant differences in frequency values obtained with the two and the three-dimensional embedded formulation are observed. Numerical and experimental results show good agreement, with a maximum difference of $7 \%$.

Larger droplets have lower values of oscillation frequency, as already reported in 39 and $[89$. Results also show that in the limit case, a zero value of frequency is achieved by an infinitely large drop 89]. Fig. 18(d) also shows numerical results obtained with the semianalytical model presented in 62 . The semi-analytical model estimates frequencies that are in reasonable agreement with computational and experimental observations thereby further validating the model compared to the results of Esposito et al. 39 which reported much higher frequencies. Even though the predictions are in relatively good agreement, the semi-analytical model is not able to provide the degree of accuracy obtained by the numerical model, especially as the volume of the droplet increases. The semi-analytical model assumes a predefined shape and does not consider gravitational effects. The former might be responsible for the discrepancies for small droplet volumes while the latter may be the cause of the differences for larger droplets.

It is important to note that frequency of oscillation does not depend on air velocity. This was stated and 89 and was confirmed in 61.

\subsubsection{Sessile droplet in a channel}

At higher current densities, fuel cell channels are particularly prone to insufficient water evacuation. Droplets with heights greater than $25 \%$ of the channel height, or even slugs, may form and eventually block the channel. 
Table 2 Water and air properties used in the simulations, considering $T=298 \mathrm{~K}$ and $p=1$ atm

\begin{tabular}{lccc}
\hline Variable & Symbol & Value & Units \\
\hline \hline Surface tension coefficient & $\gamma$ & 0.072 & $\mathrm{~N} \mathrm{~m}^{-1}$ \\
Water density & $\rho_{\mathrm{w}}$ & 1000 & $\mathrm{~kg} \mathrm{~m}^{-3}$ \\
Water viscosity & $\mu_{\mathrm{w}}$ & $10-3$ & $\mathrm{~kg} \mathrm{~m}^{-1} \mathrm{~s}^{-1}$ \\
Air density & $\rho_{\text {air }}$ & 1.205 & $\mathrm{~kg} \mathrm{~m}^{-3}$ \\
Air viscosity & $\mu_{\text {air }}$ & $1.98 \times 10^{-5}$ & $\mathrm{~kg} \mathrm{~m}^{-1} \mathrm{~s}^{-1}$ \\
\hline
\end{tabular}

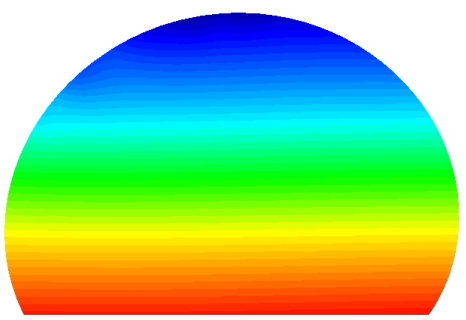

(a) Pressure profile at steady-state

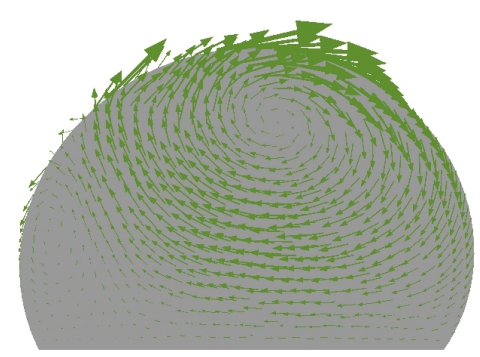

(b) Velocity field at steady-state
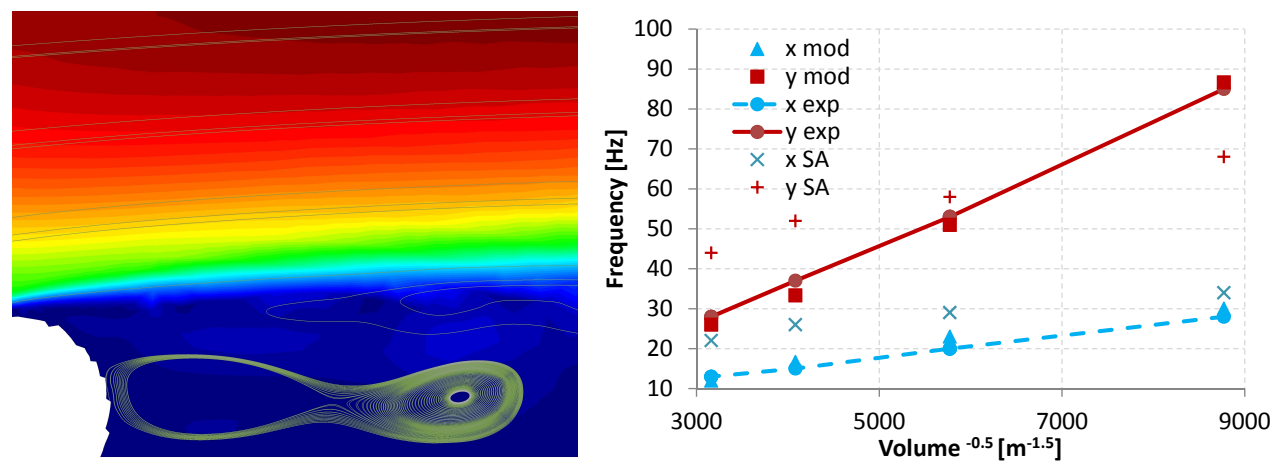

(c) Velocity profile and streamlines on the (d) Oscillation frequency vs. inverse of square root wake side of the droplet of droplet volume

Fig. 18 (a)-(b) Pressure profile and velocity field at steady-state, (c) Streamlines showing vortex formation at the wake side of the droplet and (d) Oscillation frequency versus the inverse of the square root of droplet volume, according to experiments (exp), numerical model (mod) and semi-analytical model (SA). Reproduced from 61] with kind permission of the copyright owner

Table 3 Parameters used in the sessile droplet in a free space example

\begin{tabular}{lccc}
\hline Variable & Symbol & Value & Units \\
\hline \hline Static contact angle & $\theta_{\mathrm{S}}$ & 135 & $\mathrm{deg}$ \\
Initial air velocity & $\mathbf{v}_{\text {air }}^{0}$ & 1 & $\mathrm{~m} \mathrm{~s}^{-1}$ \\
Steady-state air velocity & $\mathbf{v}_{\text {air }}^{s t}$ & 6 & $\mathrm{~m} \mathrm{~s}^{-1}$ \\
Channel height & $H$ & 50 & $\mathrm{~mm}$ \\
Channel width & $W$ & 50 & $\mathrm{~mm}$ \\
Channel length & $L$ & 500 & $\mathrm{~mm}$ \\
\hline
\end{tabular}

Thus, droplet-to-channel height ratio plays an important role on droplet oscillation and posterior detachment. Another major factor is airflow velocity. Present example studies the effects of these variables on droplet detachment by comparing the results obtained using four different approaches, i.e., experiments, two simplified (analytical) models and the embedded formulation). Results on droplet detachment are obtained with the semi-analytical model from reference 62 and the embedded model, and compared to results from Cho et al. 23. In their work, Cho et al. performed an ex-situ experimental study of droplet dynamics in a rectangular gas channel and compared experimental observations with a simplified model developed by the same authors 22 .

For droplet diameters ranging from 0.6 to $1 \mathrm{~mm}$, simulations have been performed at varying air velocities and the critical value for detachment has been obtained. The computational domain is a rectangular channel with constant cross-section. Boundary condi- 
Table 4 Parameters used in the sessile droplet in a channel example

\begin{tabular}{lccc}
\hline Variable & Symbol & Value & Units \\
\hline \hline Static contact angle & $\theta_{\mathrm{S}}$ & 128 & $\mathrm{deg}$ \\
Channel height & $H$ & 1 & $\mathrm{~mm}$ \\
Channel width & $W$ & 1.6 & $\mathrm{~mm}$ \\
Channel length & $L$ & 40 & $\mathrm{~mm}$ \\
\hline
\end{tabular}

tions coincide with the example presented in Section 4.4.1. Channel size as well as static contact angle of the substrate are detailed in Table 4. For a sessile droplet in a GDL treated with Teflon, Cho et al. 23] observed that detachment occurs when contact angle hysteresis is greater than $40 \mathrm{deg}$ [23. Therefore, this value is taken as a threshold condition for droplet detachment. Simulations with both semi-analytical and embedded models have been performed with a time step $\delta t=10^{-4} \mathrm{~s}$.

Fig. 19(a) shows the comparison between results obtained with the aforementioned four methods. Results are consistent for the different techniques: higher values of critical air velocity are obtained for smaller droplet sizes. This result is consistent with previous works [62, 120], 137], 43. However, results obtained with models exhibit more stability than those obtained experimentally. Results of the embedded model show good agreement both with numerical and experimental data from Cho et al. 23. Cho's analytical model however overpredicts critical air velocities for droplet detachment. Results obtained with the semi-analytical model of 62 show reasonable agreement with experimental data, although values for critical air velocity are underpredicted for smaller droplet diameters.

A comparison between predicted detachment times for a sessile droplet in a PEFC channel using the aforementioned methods has also been performed. Cho et al. 23 did not report detachment times in their experimental study. Let us consider the results shown in Fig. 19(a), Considering a PEFC operating at $1 \mathrm{~A} \mathrm{~cm}^{-2}$ of current density, the water injection rate in the channel is $\dot{Q}=0.1 \mu \mathrm{l} \mathrm{s}^{-1}$. Using this water injection rate and the critical droplet size for detachment, detachment time can be estimated. A comparison between the analytical model presented in 23] and the embedded and semi-analytical models is included in Table 5 .

For the embedded model, values of time and area coverage have been obtained using detachment velocity results from Fig. 19(a), In order to obtain these values for the analytical and semi-analytical models, the critical diameters for detachment used in Fig. 19(a) have been taken first. Then, for each droplet size, the corresponding value of critical velocity has been extracted from the plot in Fig. 19(a) For this velocity, the critical droplet size for each method can be directly read from the mentioned plot.

Detachment times are in agreement between the models, specially for droplets with diameters below 0.8 $\mathrm{mm}$. The semi-analytical model predicts sooner detachment values due to underpredicted detachment velocities (Fig. 19(a)). On the other hand, for droplets sizes above $1 \mathrm{~mm}$, Cho et al. predict channel blockage for velocities below $4.5 \mathrm{~m} \mathrm{~s}^{-1}$.

Droplet deformation state and velocity profile prior to detachment obtained with the embedded model for droplet sizes of 0.6 and $1 \mathrm{~mm}$ are displayed in Figs. 20(a) and 20(b) respectively. It can be observed that velocity for the bigger droplet is lower than that for the smaller droplet, as already shown in Fig. 19(a). The main difference between the $0.6 \mathrm{~mm}$-diameter and the $1 \mathrm{~mm}$ one is the vertical deformation. Whereas the former is mainly deformed in the horizontal direction, vertical deformation is also important in the latter due to channel effects [61]. This state of vertical deformation may lead to film formation in the gas channel.

The evolution of advancing and receding angles for the $0.7 \mathrm{~mm}$-diameter droplet in the simulation is shown in Fig. 19(b) The rate of change of contact angles is not constant. Three regions can be distinguished in Fig. 19(b) during the deformation process: I) both advancing and receding angles increase at the same rate, II) receding angle is constant, and III) advancing contact angle is constant, whereas receding contact angle decreases. In zone $I$, as air starts flowing around the droplet, pressure and viscous forces deform the droplet in the vertical direction. Increasing airflow velocity leads to higher pressure drop across the droplet, leading to a constant receding angle first (zone $I I$ ) and eventually diminishing, i.e., zone III. Same trends in contact angle evolution were observed experimentally by $\mathrm{Wu}$ and Djilali 129 .

Although analytical and semi-analytical offer an accurate solution to the problem at hand, they have several limitations. For instance, critical variables to understand detachment phenomena, such as airflow or droplet pressure profiles, cannot be obtained. If a detachment study is performed using two or more droplets, the two-way interactions between air and water are too complex to be described analytically. Moreover, if the droplet deforms into a film, the geometry of the domain can be hardly described by an analytical model.

For instance, using the embedded formulation, velocity and pressure profiles of the $1 \mathrm{~mm}$-diameter droplet can be directly obtained. Fig. 21 shows these variables for two time instances: at the beginning of the simulation and before droplet detachment. Vortex formation at the wake of the droplet can be observed, as already 


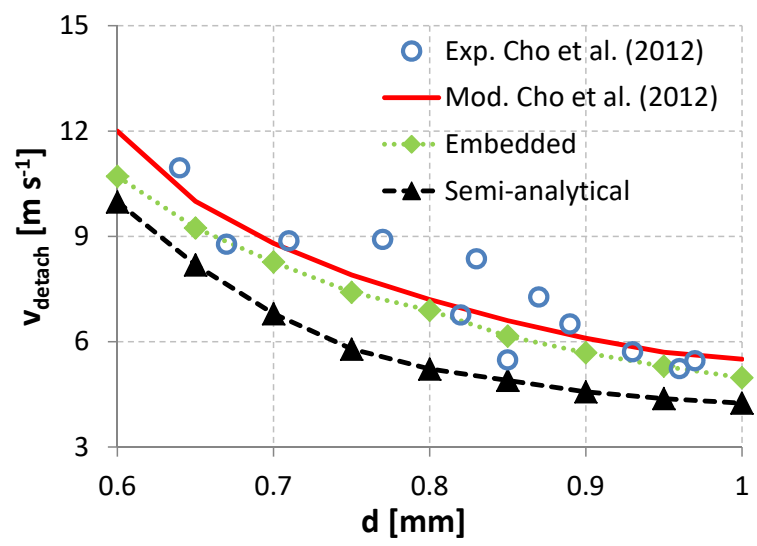

(a) Critical air velocity vs drop volume

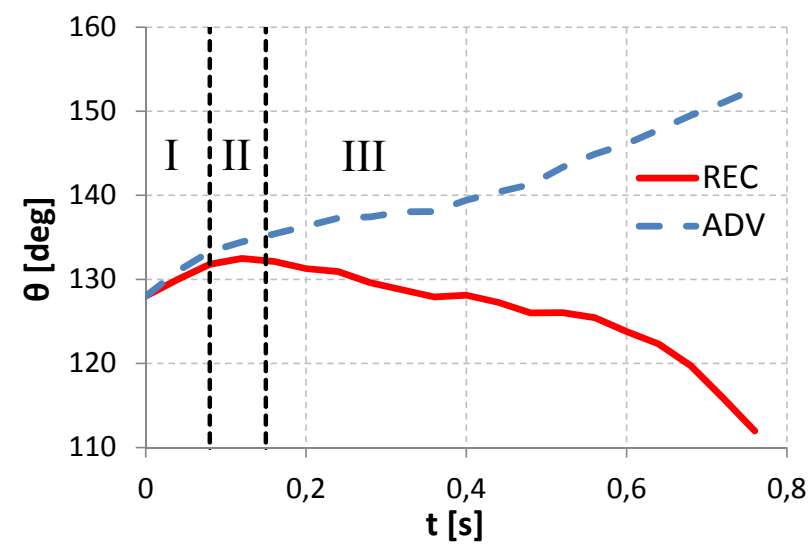

(b) Contact angle evolution

Fig. 19 (a) Critical air velocity for detachment as a function of droplet diameter obtained with analytical model from 22 (red line), experimental data from 23] (blue circle markers), the embedded model (green diamond markers) and the semi-analytical model (black triangle markers) and (b) Advancing (blue dashed line) and receding (red solid line) angle evolution for the 0.7 mm-diameter droplet

Table 5 Detachment time $\left(\mathrm{t}_{\text {det }}\right)$ and area covered $\left(\mathrm{A}_{\mathrm{cov}}\right)$ for different droplet heights $(\mathrm{h})$ according to current model and semi-analytical model presented in 62

\begin{tabular}{c|c|c|c|c|c|c}
\hline \multirow{2}{*}{$\mathrm{d}[\mathrm{mm}]$} & \multicolumn{2}{|c|}{ Embedded } & \multicolumn{2}{c}{ Semi-analytical } & \multicolumn{2}{c}{ Cho et al. } \\
\cline { 2 - 7 } & $\mathrm{t}_{\text {det }}[\mathrm{s}]$ & $\mathrm{A}_{\text {cov }}\left[\mathrm{mm}^{2}\right]$ & $\mathrm{t}_{\text {det }}[\mathrm{s}]$ & $\mathrm{A}_{\text {cov }}\left[\mathrm{mm}^{2}\right]$ & $\mathrm{t}_{\text {det }}[\mathrm{s}]$ & $\mathrm{A}_{\text {cov }}\left[\mathrm{mm}^{2}\right]$ \\
\hline \hline 0.6 & 1.02 & 0.107 & 1.24 & 0.122 & 0.88 & 0.097 \\
0.7 & 1.62 & 0.146 & 1.92 & 0.163 & 1.24 & 0.122 \\
0.8 & 2.42 & 0.190 & 2.71 & 0.205 & 1.56 & 0.142 \\
0.9 & 3.45 & 0.241 & 4.69 & 0.296 & 2.0 & 0.168 \\
1.0 & 4.73 & 0.298 & 6.3 & 0.360 & 2.42 & 0.190 \\
\hline
\end{tabular}

shown in Fig. 18(c), However, there is a difference between velocity distributions in these cases: due to channel effects, air re-attaches the channel surface in a relatively small distance compared to the case shown in Fig. 18(c). Therefore, droplet oscillation and deformation behaviors will be significantly different, confirming that droplet-to-channel height ratio is a critical variable in droplet dynamics in fuel cell channels.

\subsubsection{Droplet injection in a channel}

Water in fuel cell channels emerges from the GDL pores and either evaporates or is transported through the gas channel depending on the operating conditions of the device. It is therefore vital to characterize and study the process of emergence of liquid water into a gas channel. Droplet growth, deformation and detachment in fuel cell channels has been studied numerically 138 and experimentally 12,129] in literature. Several simulations of droplet injection in a channel have been perform in order to compare results obtained with the embedded formulation with results available in literature.
A rectangular channel with constant cross-section is considered, with a circular pore placed in the center of it, where water is injected. The same boundary conditions than those presented in Section 4.4.1 are considered with an additional Dirichlet boundary condition for water velocity at the pore. Channel size, static contact angle, inlet velocity of water and initial size of the droplet domain are detailed in Table 6. Simulations with the embedded model have been performed with a time step $\delta t=10^{-6}$ s. A comparison between numerical results and experimental data from $\mathrm{Wu}$ and Djilali [129] is presented first. Although dimensions of the channel were somewhat different from the ones typically encountered in PEFCs, results provided valuable information on droplet deformation and detachment.

A special algorithm was implemented within the embedded formulation to simulate the process of water injection from the GDL pore into the channel. This algorithm is referred as Lagrangian inlet process. For airflow in fixed Eulerian grids this condition is simply given by a prescribed velocity, i.e., Dirichlet boundary condition, at the inlet nodes. In a moving Lagrangian 


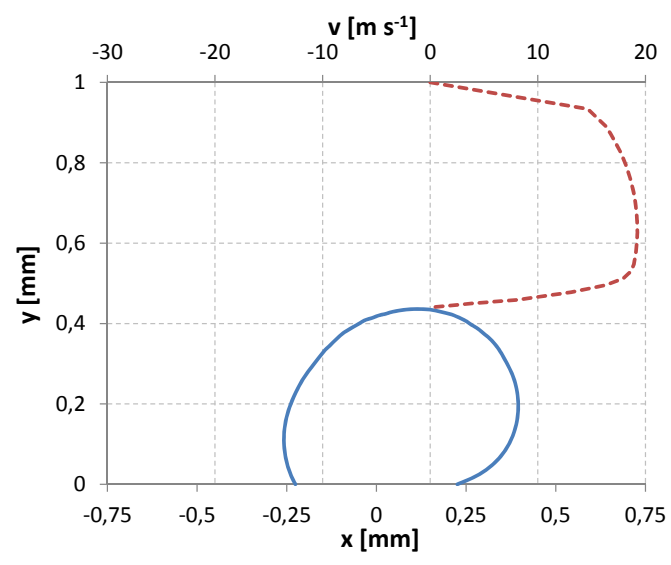

(a) $d_{\text {drop }}=0.60 \mathrm{~mm}$

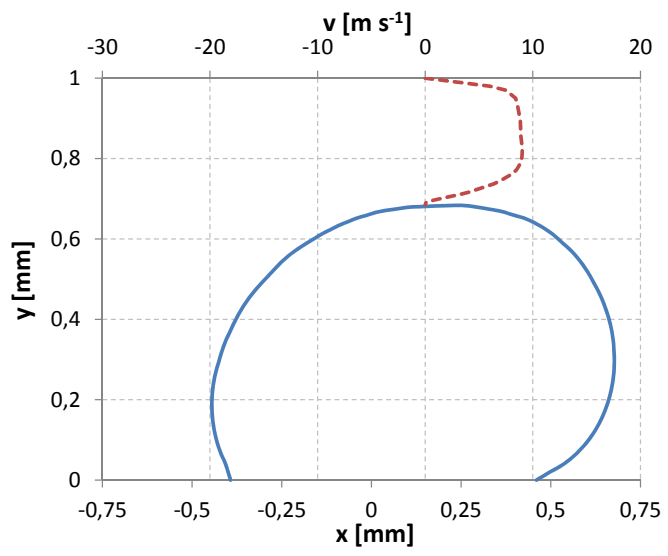

(b) $d_{\text {drop }}=1.00 \mathrm{~mm}$

Fig. 20 Drop (blue solid line) and air velocity (red dashed line) profiles at detachment in a PEFC channel

Table 6 Parameters used in the droplet injection in a channel example

\begin{tabular}{lccc}
\hline Variable & Symbol & Value & Units \\
\hline \hline Channel height & $H$ & 250 & $\mu \mathrm{m}$ \\
Channel width & $W$ & 250 & $\mu \mathrm{m}$ \\
Channel length & $L$ & 3 & $\mathrm{~mm}$ \\
Pore diameter & $D$ & 50 & $\mu \mathrm{m}$ \\
Droplet initial chord & $c$ & 100 & $\mu \mathrm{m}$ \\
Water inlet velocity & $\mathbf{v}_{\mathrm{w}}^{0}$ & 0.04 & $\mathrm{~m} \mathrm{~s}^{-1}$ \\
Air velocity & $\mathbf{v}_{\text {air }}^{0}$ & 10 & $\mathrm{~m} \mathrm{~s}^{-1}$ \\
Static contact angle & $\theta_{\mathrm{S}}$ & 110 & $\mathrm{deg}$ \\
\hline
\end{tabular}

mesh however new nodes must be injected in the vicinity of the boundary in order to represent an inlet boundary condition. More details on the Lagrangian inlet process can be found in reference 109 .

Fig. 22 shows a comparison between the experimental observations according to $\mathrm{Wu}$ and Djilali 129 and the numerical results of the embedded model for contact angle evolution. The same three regions observed in

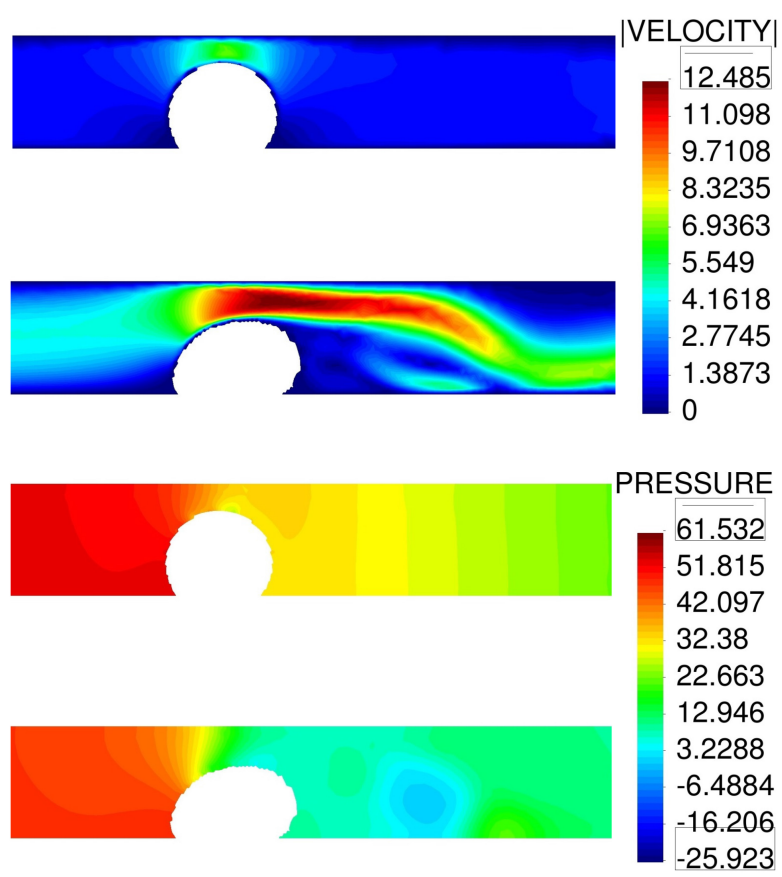

Fig. 21 Velocity (top) and pressure (bottom) distributions at the beginning of the simulation and prior to detachment for a $1 \mathrm{~mm}$-diameter droplet

Fig. 19(b) have been obtained. Numerical results agree with experimental data in zones I and II, but there is a discrepancy in the predicted receding contact angle in zone III. According to the embedded formulation, the droplet is less deformed, which can affect detachment predictions. The difference between numerical and experimental observed angles is probably due to deformation in the $z$ direction. A 3D simulation with this model is therefore needed and will be studied in the near future.

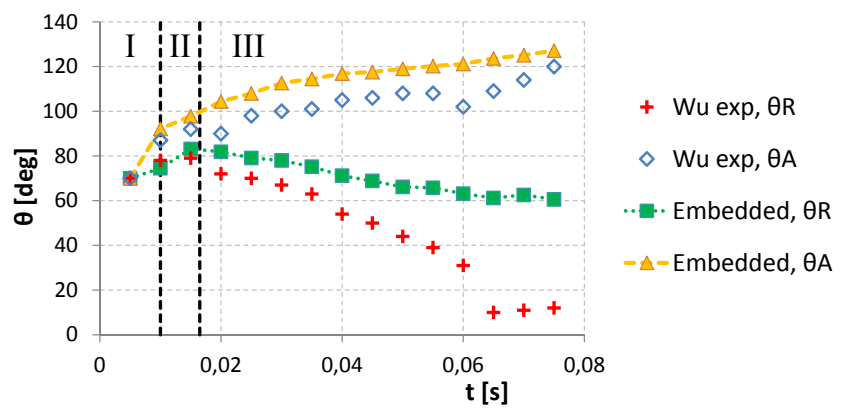

Fig. 22 Advancing (blue diamond markers) and receding (red cross markers) contact angles observed in [129], and predicted advancing (orange triangle markers) and receding (green square markers) contact angles with the embedded formulation. Reproduced from 109 with kind permission of the copyright owner 
A similar setup was used for the computational domain in the numerical study presented by Zhu et al. 137, the only difference being that the injection pore was placed at $250 \mu \mathrm{m}$ from the channel inlet. Instead of $0.04 \mathrm{~m} \mathrm{~s}^{-1}$, water was injected at a constant velocity of $0.1 \mathrm{~m} \mathrm{~s}^{-1}$. The time step used in the simulations with the embedded model is $\delta t=10^{-6} \mathrm{~s}$, whereas in 137 this parameter was set to $\delta t=10^{-7} \mathrm{~s}$.

Comparison between result obtain with VOF and embedded models is displayed in Fig. 23. According to the embedded formulation, the droplet quickly deforms and elongates along-the-channel direction, forming a film. However, results obtained by Zhu et al. show a less deformed droplet, with areas of the droplet where no surface curvature is observed $(\mathrm{t}=2 \mu \mathrm{s})$. These results seem to be non-physical since the droplet is injected near the channel inlet, and both airflow and water injection velocities are high. Therefore, film formation under these conditions is more realistic rather than a vertically-elongated droplet. No experimental validation of this example was provided by Zhu et al [137.

\subsubsection{Computational efficiency}

For the example presented in Section 4.4.1, the embedded and semi-analytical models have been used to study the effects of the droplet volume in its oscillations. In both cases, the time step used has been $\delta t=10^{-3} \mathrm{~s}$ for a simulation of $1 \mathrm{~s}$. Results have been obtained relatively quick with the embedded formulation (less than $30 \mathrm{~min}$ on a desktop Intel i5 quad-core PC), the semi-analytical model however is capable of performing the simulations in less than 2 min. Although the embedded model gives better predictions of droplet oscillation, this example manifests the advantage of the semi-analytical model for a simple case.

Both embedded and semi-analytical models were used again in Section 4.4.2 to predict the critical velocity to detach a sessile droplet in a channel. In this case, the time step has been reduced on order of magnitude, i.e., $\delta t=10^{-4} \mathrm{~s}$. The computational times were 5 min for the semi-analytical model and less than 1 hour for the embedded method. Although simulations are more computationally intensive with the latter method, predictions for detachment have better agreement with experimental data from 23]. The analytical model developed by Cho et al. was steady-state, and no computational times were reported in their study 23 .

The example in Section 4.4.3 is more computationally demanding and therefore the time step used is further reduced. A time step of $\delta t=10^{-6} \mathrm{~s}$ is used in the embedded formulation, whereas Zhu et al. 137] report a value of $\delta t=10^{-7} \mathrm{~s}$. The computational time for the embedded formulation was 4 hours, approximately. No details on computational cost were given by Zhu et al. 137.

Overall, the semi-analytical model has proven to be a fast model for the analysis of sessile droplets in channels, performing approximate predictions in less than 5 minutes. However, in some cases (e.g. when gravity effects are not negligible) the model fails to give accurate results. On the other hand, the embedded formulation is capable of predicting droplet oscillation and detachment behavior efficiently, with time steps (at least) one order of magnitude higher than other studies in literature.

\subsubsection{Conclusions drawn from applying the embedded model to the problem at hand}

Results have shown that for analysis of liquid transport in gas channels viscous effects of the airflow upon the droplets are essential. Therefore, it is essential to couple the entire stress (i.e., pressure and deviatoric part) between the liquid and gas sub-domains. Results of the embedded models were consistent with previous works, and good agreement was obtained with experimental data available [23, 89, 129]. Effects of droplet size on detachment velocity and time have been also studied. Smaller droplets exhibit higher values of air velocity needed for detachment. Reasonable agreement between results and experimental data found in literature was obtained 23.

Water injection in a gas channel has been studied and results have been compared to previous numerical 137 and experimental results 129. Excellent agreement between experimental observation and numerical results has been obtained for early stages of droplet deformation. Advancing contact angle increases approximately at a constant rate. On the other hand, receding angle increases during a short period of time, followed by a transition zone where receding angle remains constant. As the droplet volume increases, pressure and viscous effects deform the droplet in longitudinal direction of the channel, and receding constant angle decreases until detachment was observed. Numerical results predict a lower rate of diminishing than experimental results. This discrepancy may be due to effects in cross-channel direction.

The embedded formulation has certain advantages compared to fixed-grid methods. For sessile drop studies where only gravity effects on deformation are analyzed with air in quiescent conditions (e.g. Example 1 in [61]) effects of ambient air on droplet deformation are negligible. Thus, these studies can be performed only by solving the liquid domain, increasing the efficiency of 


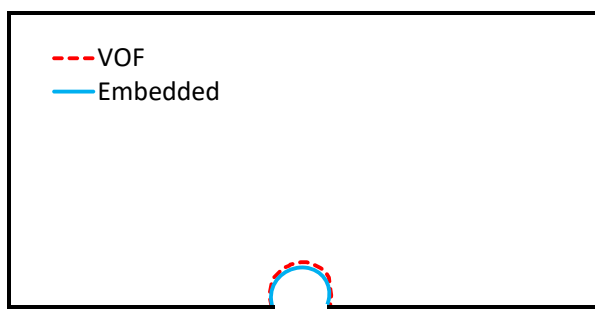

(a) $t=0.4 \mathrm{~ms}$

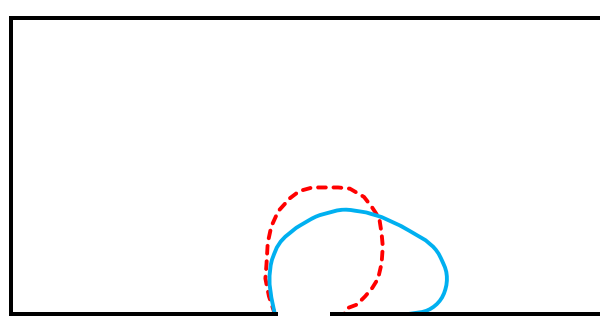

(c) $t=2 \mathrm{~ms}$

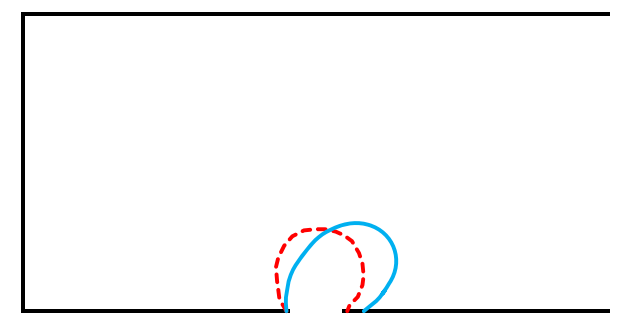

(b) $t=1 \mathrm{~ms}$

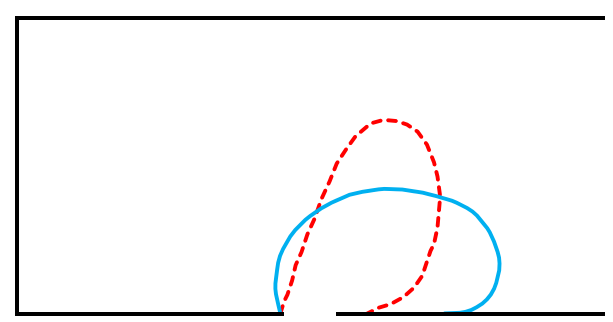

(d) $t=3.3 \mathrm{~ms}$
Fig. 23 Numerical results according to VOF model from 137 deformation process of an emerging droplet into a gas channel.

the method. Unlike the embedded model, the option of "turning off" the solution of the surrounding gas domain is not possible in fixed-grid meshes. This feature of the embedded formulation may be also advantageous for the simulation of the liquid transport through the GDL, as it would allows to include only the "wet" part of the GDL in the computation.

It was recently shown by Jarauta et al. 60. that the convergence of the non-linear procedure for the embedded formulation is linear. The same order of convergence was obtained for a gravity-driven problem and for a surface tension-dominated problem. Therefore, the order of convergence is intrinsic of the method and does not depend on the problem solved.

\section{Summary and conclusions}

This paper presented an overview of techniques used for studying two-phase flow in fuel cell gas channels. Among the computational models two types were presented in detail:

- Analytical ("force balance") models: approaches capable of predicting droplet detachment condition in gas channels for droplet flow regime at a reduced computational cost.

- Two-phase CFD models: approaches representing the interaction of the liquid and the gas phases using continuum mechanics hypothesis. These are charac- terized by higher accuracy and elevated computational cost.

The analytical models are based on a net force balance applied to an a priori known (or assumed) droplet geometry. These models can be used to predict the detachment times (in case of dynamic models) or detachment conditions (in case of steady-state simulations). Usually, the detachment conditions are determined as the ones resulting in a critical value contact angle hysteresis, which characterizes a given GDL material and is known from experiments.

In the most recent work 62 the force balance on the droplet has been modeled using an analytic expression, but integrated numerically at every time step on an approximate droplet geometry. It was discovered that computing the drag coefficient numerically, rather than directly using the analytic expression for the given shape leads to a considerable improvement in the accuracy. Moreover, it was concluded that when the droplet height increases, the classical analytic expression for the drag force (linearly dependence on the drag coefficient and quadratic dependence on the air velocity) does not provide acceptable results as the hypothesis of the droplet under a free air stream is violated. A critical droplet height to channel height ratio was identified as approximately 10\%. Above this value the drag force shows a nonlinear relationship with the Reynolds number and the contact angle hysteresis.

The differences between the models based on the force balance and the experimental results become more 
evident for higher droplet volumes. Even the most recent semi-analytical model tends to under-predict detachment velocities in such cases. On the other hand, for small droplets, where gravity effects are negligible, the semi-analytical model can be considered a valuable and efficient tool for preliminary estimation of droplets detachment. Nevertheless, all the "force balance" (analytical and semi-analytical) models are not able to deal with strongly deforming droplets, growing droplets, perform multiple droplets analysis or predict post-detachment behavior. They exclude the possibility of including the feedback of the liquid phase onto the airflow, an effect that may not be negligible particularly for large droplets. Moreover, liquid phase injection from the GDL, surface roughness effects cannot be accounted for. None of the flow regimes, other than the droplet one cannot be analyzed.

In order to overcome these drawbacks, numerical models based on coupled solution of the liquid and gas phases under assumptions of continuum mechanics have been applied. Among the existing two-phase models one may distinguish the volume of fluid approaches that solve the two-phase problem on a single fixed (Eulerian) mesh and the more recent embedded Eulerian-Lagrangian models. The practical advantage of volume of fluid model consists in the fact that they are available in general-purpose commercial (such as Ansys FLUENT) and open source (e.g. OpenFOAM) software. VOF simulations provided valuable data for multiple flow channel configurations and flow conditions. Nevertheless, VOF simulations of the problem at hand carried out to date were generally characterized by large computational costs due to a) explicit integration of surface tension force, b) necessity of using very fine meshes to capture the liquid-gas interface, and c) monolithic solution of two phases. These factors result in a very small value of critical time step necessary for obtaining stable solutions. Overall, one can conclude that VOF models must be adapted to the problem at hand in order to obtain more efficient implementations. In particular, improved surface tension models must be sought in order to ensure unconditional stability and thus of increase critical time steps. Implicit surface tension models for fixed grid approaches such as 56, 116, 118 must be considered by the PEFC modeling community.

On the other hand, the recently developed embedded Eulerian-Lagrangian model was found particularly advantageous for the problem at hand. It efficiently partitions the problem into gas and liquid sub-problems, which allows using best-suitable models for the individual phases coupled in a straight-forward way using a simple Dirichlet-Neumann coupling. An important ad- vantage of the proposed embedded approach is that the liquid-gas interface position is accurately defined by the Lagrangian mesh. Thus, no additional techniques (typically used in fully Eulerian formulations) for interface tracking were necessary. The strong discontinuity of pressure across the air-water interface due to surface tension effects is accounted for naturally in the embedded models. The model allowed for using larger time steps in comparison with the formerly used approaches due to an implicit surface tension model proposed, leading to a considerable improvement in computational efficiency. A dynamic contact angle condition proposed in the embedded model allowed to accurately reproduce wetting dynamics on GDL. A good agreement between the numerical results and the experiments approve that the embedded model defines a viable alternative to the VOF models.

\subsection{Future developments}

All the models mentioned in this work were applied to analysis of a single droplet or a few ones emerging from idealized GDL pores at hypothetic locations considered known a priori. In reality water emerges through multiple "preferential locations" pores that may vary in the transient case $[12$. Ideally, these pores must be determined by a liquid transport simulation applied to the GDL.

Recent advances in the image-based reconstruction allowed for obtaining the actual structures of the diffusive media (X-rays, SEM/TEM microscopy) [58], [71. These images open new frontiers as they can provide the geometrical basis for "direct numerical simulations 4 on micro-scale meshes 110. However, such pore scale simulations are extremely tedious computationally and thus cannot be used for industrial purposes. Thus, one interesting possibility for the future research is to develop a multi-scale model to simulate the entirety of the GDL using the equations for the flow through porous media 127. The different factors influencing the water flow, such as material composition, saturation of pores or pre-existing water can be introduced into the model by modifying the effective permeability of the media dynamically at each point in space. The non-linearities inherent in the small-scale problem will be therefore represented by the local value chosen for the permeability.

To ensure that such a model provides an accurate representation of the flow over the porous layer, one can use deep learning techniques (neural networks-based)

4 The term "direct numerical simulation" is used here in the sense of explicitly resolving the micro-scale 
[14, [48 to obtain a relationship between the conditions of the flow, the geometry and material properties of the diffusion media and the effective permeability of the material. To do this, a series of small-scale simulations must be carried out using actual structure of the diffusion media extracted from the high resolution images. Fig. 24 shows the conceptual scheme of such multiscale model. The multi-scale model must be also capable of "translating" the effective properties into boundary conditions for the macro-scale simulation (see 35. for such coupling in a different context) in gas channel (location and size of injection sites).

Ultimately, the boundary conditions such as liquid injection rate and airflow velocity are typically considered constant input parameters in PEFC two-phase simulations. Nevertheless, the non-uniform reaction at catalyst layer results in a non-uniform rate of liquid water generation. Partial water accumulation, in turn, affects the diffusion of reactants and thus the reaction rates. Thus, it is of essential importance to couple the mechanical and electrochemical computational modules in order to represent realistic operation conditions. Open source codes coupling defines, most probably, the only possibility of bringing together the knowhow developed in fields necessary for representing the overall fuel cell behavior.

Acknowledgements This work has been supported by the Natural Science and Engineering Research Council of Canada (NSERC) Collaborative Research and Development grant NSERC CRDPJ 445887-12, the FPI Research Grant BES2011-047702 subject to the Spanish Project BIA2010-15880, and the Jose Castillejo grant (ref. CAS17/00184) from the Ministerio de Eduaciín, Cultura y Deporte of Spain.

$$
\begin{array}{ll}
\mathbf{v} & \text { velocity, }\left[\begin{array}{ll}
\mathrm{m} & \mathrm{s}^{-1}
\end{array}\right] \\
B & \text { Channel's half height, }[\mathrm{m}] \\
C_{k} & \text { volume fraction of fluid } k,[-] \\
p & \text { pressure, }\left[\begin{array}{ll}
\mathrm{N} & \mathrm{m}^{-2}
\end{array}\right] \\
t & \text { time, }[\mathrm{s}] \\
U & \text { Mean airflow velocity, }\left[\begin{array}{ll}
\mathrm{m} & \mathrm{s}^{-1}
\end{array}\right]
\end{array}
$$

\section{Abbreviations}

ALE arbitrary Lagrangian/Eulerian

CFD computational fluid dynamics

CL catalyst layer

CSF continuum surface force

CSS continuum surface stress

GDL gas diffusion layer

HOR hydrogen oxidation reaction

MEA membrane electrode assembly

ORR oxygen reduction reaction

PEFC polymer electrolyte fuel cells

PTFE polytetrafluoroethylene

SUPG streamline upwind Petrov-Galerkin

VOF volume of fluid

\section{Greek letters}

$\boldsymbol{\sigma}$ Cauchy stress tensor, $\left[\begin{array}{ll}\mathrm{N} & \mathrm{m}^{-2}\end{array}\right]$

$\Delta \quad$ contact angle hysteresis, [rad]

$\gamma \quad$ surface tension coefficient, $\left[\begin{array}{ll}\mathrm{N} & \left.\mathrm{m}^{-1}\right]\end{array}\right.$

$\kappa \quad$ surface curvature, $\left[\mathrm{m}^{-1}\right]$

$\mu \quad$ dynamic viscosity, $\left[\begin{array}{lll}\mathrm{kg} & \mathrm{m}^{-1} & \mathrm{~s}^{-1}\end{array}\right]$

$\nu \quad$ kinematic viscosity, $\left[\begin{array}{ll}\mathrm{m}^{2} & \mathrm{~s}^{-1}\end{array}\right]$

$\rho$ density, $\left[\begin{array}{ll}\mathrm{kg} & \mathrm{m}^{-3}\end{array}\right]$

$\tau \quad$ algorithmic stabilization parameter, [-]

$\theta \quad$ contact angle, [rad]

$\theta_{A} \quad$ advancing contact angle, $[\mathrm{rad}]$

$\theta_{R} \quad$ receding contact angle, $[\mathrm{rad}]$

$\theta_{S} \quad$ static contact angle, [rad]

\section{Mathematical operators}

$\nabla_{s} \quad$ tangential gradient operator

\section{Nomenclature}

$\overline{\mathbf{F}} \quad$ body force vector, $\left[\mathrm{N} \quad \mathrm{m}^{-3}\right]$

G gradient matrix, [-]

L Laplacian matrix, [-]

$\overline{\mathbf{p}} \quad$ nodal pressure, $\left[\mathrm{N} \mathrm{m}^{-2}\right]$

I unit tensor, [-]

D divergence matrix, [-]

$\mathbf{H}$ tangent matrix, [-]

K convection stabilization matrix, [-]

M mass matrix, [-]

m unit tangent vector to the contact line, [-]

$\mathbf{N} \quad$ velocity shape function, [-]

$\mathbf{n} \quad$ unit normal vector, [-]

$\mathbf{N}_{p} \quad$ pressure shape function, [-]

$\mathbf{S}$ pressure stabilization matrix, [-]

$\mathbf{t} \quad$ unit tangent vector, [-]

$\overline{\mathbf{v}} \quad$ nodal velocity, $\left[\begin{array}{ll}m & \mathrm{~s}^{-1}\end{array}\right]$

\section{References}

1. ANSYS CFX. http://www.ansys.com/products/fluids/ ansys-cfx

2. ANSYS Fluent. http://www.ansys.com/Products/ Fluids/ANSYS-Fluent

3. COMSOL Multiphysics. https://www. comsol.com/

4. CSC - Elmer. https://www.csc.fi/web/elmer

5. OpenFOAM. http://www.openfoam.com/

6. OpenFVM. http://openfvm. sourceforge.net/

7. Computational Fluid Dynamics of Water Droplet Formation and Detachment from Gas Diffusion Layer. proceedings of 221st Electrochemical Society (ECS) Meeting (2012)

8. Ahmad, Z., Didari, S., Moon, J., Harris, T.: Computational fluid dynamics of water droplet formation and detachment from gas diffusion layer. ECS Transactions 45(23), 89-100 (2013)

9. Anderson, R., Zhang, L., Ding, Y., Blanco, M., Bi, X., Wilkinson, D.P.: A critical review of two-phase flow in 


\section{Pre-computed data \\ based on actual images \\ of the diffusion media}
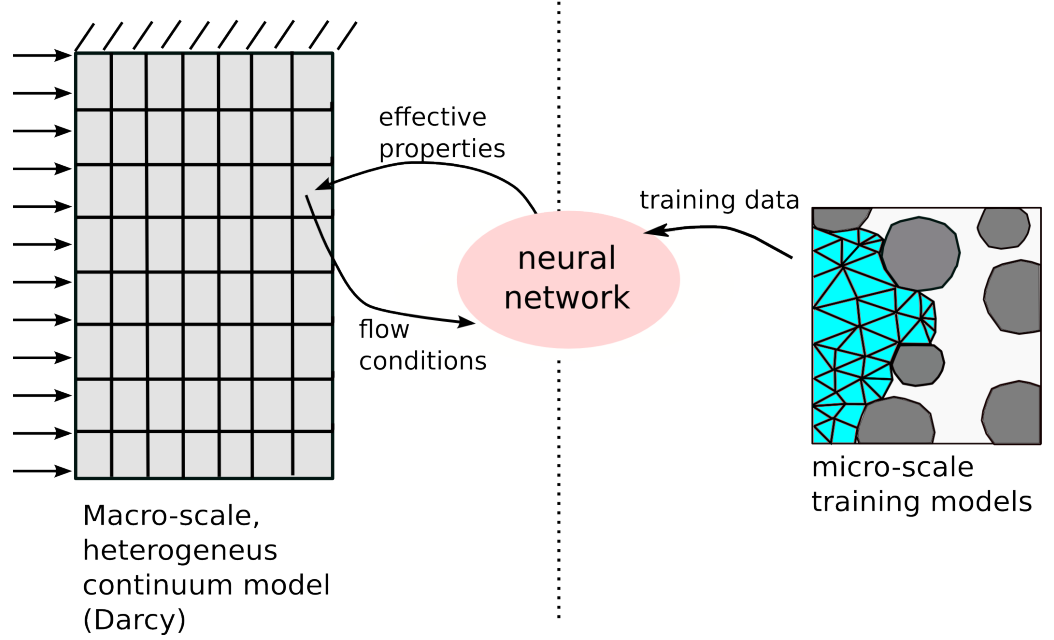

Fig. 24 The multi-scale paradigm enriched with neural network training for the simulation of liquid transport through the diffusion media.

gas flow channels of proton exchange membrane fuel cells. Journal of Power Sources 195(15), 4531-4553 (2010)

10. Ashrafi, M., Shams, M.: Effects of heterogeneous surface of gas diffusion layers on droplet transport in microchannels of pem fuel cells. International journal of hydrogen energy 41(3), 1974-1989 (2016)

11. Ashrafi, M., Shams, M., Bozorgnezhad, A., Ahmadi, G.: Simulation and experimental validation of droplet dynamics in microchannels of pem fuel cells. Heat and Mass Transfer 52(12), 2671-2686 (2016)

12. Bazylak, A., Sinton, D., Djilali, N.: Dynamic water transport and droplet emergence in PEMFC gas diffusion layers. Journal of Power Sources 176(1), 240-246 (2008)

13. Benziger, J.B., Satterfield, M.B., Hogarth, W.H., Nehlsen, J.P., Kevrekidis, I.G.: The power performance curve for engineering analysis of fuel cells. Journal of power sources 155(2), 272-285 (2006)

14. Bose, N.K., et al.: Neural network fundamentals with graphs, algorithms, and applications. 612.82 BOS (1996)

15. Brackbill, J., Kothe, D., Zemach, C.: A continuum method for modeling surface tension. Journal of Computational Physics 100, 335-354 (1992)

16. Cai, Y., Hu, J., Ma, H., Yi, B., Zhang, H.: Effects of hydrophilic/hydrophobic properties on the water behavior in the micro-channels of a proton exchange membrane fuel cell. Journal of Power Sources 161(2), 843-848 (2006)

17. Carton, J., Lawlor, V., Olabi, A., Hochenauer, C., Zauner, G.: Water droplet accumulation and motion in pem (proton exchange membrane) fuel cell minichannels. Energy 39(1), 63-73 (2012)

18. Chen, K., Hickner, M., Noble, D.: Simplified models for predicting the onset of liquid water droplet instability at the gas diffusion layer/gas flow channel interface. International Journal of Energy Research 29(12), 1113-1132 (2005)
19. Chen, L., Cao, T., Li, Z., He, Y., Tao, W.: Numerical investigation of liquid water distribution in the cathode side of proton exchange membrane fuel cell and its effects on cell performance. international journal of hydrogen energy 37(11), 9155-9170 (2012)

20. Chen, L., He, Y., Tao, W.: Effects of surface microstructures of gas diffusion layer on water droplet dynamic behaviors in a micro gas channel of proton exchange membrane fuel cells. International Journal of Heat and Mass Transfer 60, 252-262 (2013)

21. Chen, L., Luan, H., He, Y., Tao, W.: Effects of roughness of gas diffusion layer surface on liquid water transport in micro gas channels of a proton exchange membrane fuel cell. Numerical Heat Transfer, Part A: Applications 62(4), 295-318 (2012)

22. Cho, S.C., Wang, Y., Chen, K.: Droplet dynamics in a polymer electrolyte fuel cell gas flow channel: Forces, deformation, and detachment. I: Theoretical and numerical analyses. Journal of Power Sources 206, 119-128 (2012)

23. Cho, S.C., Wang, Y., Chen, K.: Droplet dynamics in a polymer electrolyte fuel cell gas flow channel: Forces, deformation and detachment. II: Comparisons of analytical solution with numerical and experimental results. Journal of Power Sources 210, 191-197 (2012)

24. Choi, J., Son, G.: Numerical study of droplet motion in a microchannel with different contact angles. Journal of mechanical science and technology 22(12), 2590-2599 (2008)

25. Choi, J., Son, G.: Numerical study of droplet dynamics in a pemfc gas channel with multiple pores. Journal of mechanical science and technology 23(7), 1765-1772 (2009)

26. Chorin, A.J.: A numerical method for solving incompressible viscous problems. Journal of Computational Physics 2, 12-26 (1967)

27. Codina, R., Houzeaux, G., Coppola-Owen, H., Baiges, J.: The fixed-mesh ALE approach for the numerical approximation of flows in moving domains. Journal of Computational Physics 228(5), 1591 - 1611 (2009) 
28. Colosqui, C.E., Cheah, M.J., Kevrekidis, I.G., Benziger, J.B.: Droplet and slug formation in polymer electrolyte membrane fuel cell flow channels: The role of interfacial forces. Journal of Power Sources 196, 10,057-10,068 (2011)

29. Daino, M.M., Lu, Z., LaManna, J.M., Owejan, J.P., Trabold, T.A., Kandlikar, S.G.: Through-plane water transport visualization in a pemfc by visible and infrared imaging. Electrochemical and Solid-State Letters 14(6), B51-B54 (2011)

30. Ding, Y., Anderson, R., Zhang, L., Bi, X., Wilkinson, D.: Simulations of two-phase flow distribution in communicating parallel channels for a PEM fuel cell. International Journal of Multiphase Flow 52, 35-45 (2013)

31. Ding, Y., Bi, H., Wilkinson, D.: Three-dimensional numerical simulation of water droplet emerging from a gas diffusion layer surface in micro-channels. Journal of Power Sources 195(21), 7278-7288 (2010)

32. Ding, Y., Bi, H., Wilkinson, D.: Three dimensional numerical simulation of gas-liquid two-phase flow patterns in a polymer-electrolyte membrane fuel cells gas flow channel. Journal of Power Sources 196(15), 6284-6292 (2011)

33. Ding, Y., Bi, X., Wilkinson, D.: 3d simulations of the impact of two-phase flow on PEM fuel cell performance. Chemical Engineering Science 100, 445-455 (2013)

34. Ding, Y., Bi, X., Wilkinson, D.: Numerical investigation of the impact of two-phase flow maldistribution on pem fuel cell performance. International Journal of Hydrogen Energy 39(1), 469-480 (2014)

35. Discacciati, M., Quarteroni, A.: Navier-stokes/darcy coupling: modeling, analysis, and numerical approximation (2009)

36. Donea, J., Huerta, A.: Finite Element Methods for Flow Problems, 1st edn. John Wiley \& Sons (2003)

37. Eller, J., Büchi, F.N.: Polymer electrolyte fuel cell performance degradation at different synchrotron beam intensities. Journal of synchrotron radiation 21(1), 82-88 (2014)

38. Ellis, M.W., Von Spakovsky, M.R., Nelson, D.J.: Fuel cell systems: efficient, flexible energy conversion for the 21st century. Proceedings of the IEEE 89(12), 18081818 (2001)

39. Esposito, A., Polverino, P., Pianese, C., Guezennec, Y.: A lumped model of single droplet deformation, oscillation and detachment on the GDL surface of a PEM fuel cell. ASME 2010 8th International Fuel Cell Science, Engineering and Technology Conference (2010)

40. Extrand, C., Kumagai, Y.: Liquid drops on an inclined plane: the relation between contact angles, drop shape, and retentive force. Journal of Colloid and Interface Science 170, 515-521 (1995)

41. Ferreira, R., Falcão, D., Oliveira, V., Pinto, A.: Numerical simulations of two-phase flow in an anode gas channel of a proton exchange membrane fuel cell. Energy 82, 619-628 (2015)

42. Fontana, E., Mancusi, E., de Souza, A., de Souza, S.: Flow regimes for liquid water transport in a tapered flow channel of proton exchange membrane fuel cells (pemfcs). Journal of Power Sources 234, 260-271 (2013)

43. Golpaygan, A., Ashgriz, N.: Effects of oxidant fluid properties on the mobility of water droplets in the channels of PEM fuel cell. International journal of energy research 29(12), 1027-1040 (2005)

44. Golpaygan, A., Ashgriz, N.: Multiphase flow model to study channel flow dynamics of PEM fuel cells: deformation and detachment of water droplets. International
Journal of Computational Fluid Dynamics 22(1-2), 8595 (2008)

45. Golpaygan, A., Sarchami, A., Ashgriz, N.: Threedimensional multiphase flow model to study channel flow dynamics of pem fuel cells. International Journal of Energy Research 35(13), 1188-1199 (2011)

46. Gostick, J.: Random pore network modeling of GDLs using Voronoi and Delaunay tessellations. ECS Transactions 41(1), 125-130 (2011)

47. Hakenjos, A., Muenter, H., Wittstadt, U., Hebling, C.: A pem fuel cell for combined measurement of current and temperature distribution, and flow field flooding. Journal of Power Sources 131, 213-216 (2004)

48. Haykin, S., Network, N.: A comprehensive foundation. Neural Networks 2(2004), 41 (2004)

49. He, G., Yamazaki, Y., Abudula, A.: The effect of wall roughness on the liquid removal in micro-channels related to a proton exchange membrane fuel cell (PEMFC). Journal of Power Sources 195(6), 1561-1568 (2010)

50. Hickner, M.A., Siegel, N.P., Chen, K.S., McBrayer, D.N., Hussey, D.S., Jacobson, D.L., Arif, M.: Real-time imaging of liquid water in an operating proton exchange membrane fuel cell. Langmuir 22(14), 6234-6237 (2006)

51. Hirt, C., Nichols, B.: Volume of Fluid (VOF) method for the dynamics of free boundaries. Journal of Computational Physics 39, 201-225 (1981)

52. Hossain, M., Islam, S., Colley-Davies, A., Adom, E.: Water dynamics inside a cathode channel of a polymer electrolyte membrane fuel cell. Renewable energy 50, 763-779 (2013)

53. Hou, Y., Zhang, G., Qin, Y., Du, Q., Jiao, K.: Numerical simulation of gas liquid two-phase flow in anode channel of low-temperature fuel cells. International Journal of Hydrogen Energy 42(5), 3250-3258 (2017)

54. Hughes, T.J., Franca, L.P., Balestra, M.: A new finite element formulation for computational fluid dynamics: V. circumventing the babuska-brezzi condition: a stable petrov-galerkin formulation of the stokes problem accommodating equal-order interpolations. Computer Methods in Applied Mechanics and Engineering 59(1), 85-99 (1986)

55. Hussaini, I.S., Wang, C.Y.: Visualization and quantification of cathode channel flooding in pem fuel cells. Journal of Power Sources 187(2), 444-451 (2009)

56. Hysing, S.: A new implicit surface tension implementation for interfacial flows. International Journal of $\mathrm{Nu}-$ merical Methods in Fluids 6(51), 659-672 (2006)

57. Idelsohn, S., Mier-Torrecilla, M., Oñate, E.: Multi-fluid flows with the particle finite element method. Computer Methods in Applied Mechanics and Engineering 198(33), 2750-2767 (2009)

58. James, J., Choi, H.W., Pharoah, J.: X-ray computed tomography reconstruction and analysis of polymer electrolyte membrane fuel cell porous transport layers. international journal of hydrogen energy $\mathbf{3 7}(23)$, 18,216$18,230(2012)$

59. Jarauta, A.: Modeling of droplet dynamics in a Proton Exchange Fuel Cell electrode channel. Ph.D. thesis, Universitat Politècnica de Catalunya (2016)

60. Jarauta, A., Ryzhakov, P.B., Secanell, M., , Pons-Prats, J.: An implicit finite element model for droplets and two-phase flows with strong surface tension effects. International Journal of Multiphase flows (2017). Submitted 
61. Jarauta, A., Ryzhakov, P.B., Secanell, M., Waghmare, P.R., Pons-Prats, J.: Numerical study of droplet dynamics in a polymer electrolyte fuel cell gas channel using an embedded Eulerian-Lagrangian approach. Journal of Power Sources 323, 201-212 (2016)

62. Jarauta, A., Secanell, M., Pons-Prats, J., Ryzhakov, P.B., Idelsohn, S.R., Oñate, E.: A semi-analytical model for droplet dynamics on the GDL surface of a PEFC electrode. International Journal of Hydrogen Energy 40, 5375-5383 (2015)

63. Jiao, K., Zhou, B.: Innovative gas diffusion layers and their water removal characteristics in pem fuel cell cathode. Journal of Power Sources 169(2), 296-314 (2007)

64. Jiao, K., Zhou, B.: Accelerated numerical test of liquid behavior across gas diffusion layer in proton exchange membrane fuel cell cathode. Journal of fuel cell science and technology 5(4), 041,011 (2008)

65. Jiao, K., Zhou, B., Quan, P.: Liquid water transport in parallel serpentine channels with manifolds on cathode side of a PEM fuel cell stack. Journal of Power Sources 154(1), 124-137 (2006)

66. Jiao, K., Zhou, B., Quan, P.: Liquid water transport in straight micro-parallel-channels with manifolds for pem fuel cell cathode. Journal of Power Sources 157, 226-243 (2006)

67. Jiao, K., Zhou, B., Quan, P.: Liquid water transport in straight micro-parallel-channels with manifolds for PEM fuel cell cathode. Journal of Power Sources 157(1), 226-243 (2006)

68. Jo, J., Kim, W.: Numerical simulation of water droplet dynamics in a right angle gas channel of a polymer electrolyte membrane fuel cell. International Journal of Hydrogen Energy 40(26), 8368-8383 (2015)

69. Kamran, K., Rossi, R., Onate, E., Idelsohn, S.: A compressible lagrangian framework for the simulation of the underwater implosion of large air bubbles. Computer Methods in Applied Mechanics and Engineering 255, 210-225 (2013)

70. Kang, S., Zhou, B., Cheng, C., Shiu, H., Lee, C.: Liquid water flooding in a proton exchange membrane fuel cell cathode with an interdigitated design. International Journal of Energy Research 35(15), 1292-1311 (2011)

71. Khajeh-Hosseini-Dalasm, N., Sasabe, T., Tokumasu, T., Pasaogullari, U.: Effects of polytetrafluoroethylene treatment and compression on gas diffusion layer microstructure using high-resolution x-ray computed tomography. Journal of Power Sources 266, 213-221 (2014)

72. Kim, H., Jeon, S., Song, M., Kim, K.: Numerical simulations of water droplet dynamics in hydrogen fuel cell gas channel. Journal of Power Sources 246, 679-695 (2014)

73. Kim, H., Nam, J., Shin, D., Chung, T., Kim, Y.: Computational fluid dynamics simulations for hydrogen dispersion and exhaust in residential fuel cell systems. Current Applied Physics 10(2), S81-S85 (2010)

74. Kumbur, E., Sharp, K., Mench, M.: Liquid droplet behavior and instability in a polymer electrolyte fuel cell flow channel. Journal of Power Sources 161, 333-345 (2006)

75. Lafaurie, B., Nardone, C., Scardovelli, R., Zaleski, S., Zanetti, G.: Modelling merging and fragmentation in multiphase flows with SURFER. Journal of Computational Physics 113(1), 134-147 (1994)

76. Le, A., Zhou, B.: A general model of proton exchange membrane fuel cell. Journal of power sources 182(1), 197-222 (2008)
77. Le, A., Zhou, B.: A generalized numerical model for liquid water in a proton exchange membrane fuel cell with interdigitated design. Journal of Power Sources 193(2), 665-683 (2009)

78. Le, A., Zhou, B.: A numerical investigation on multiphase transport phenomena in a proton exchange membrane fuel cell stack. Journal of Power Sources 195(16), 5278-5291 (2010)

79. Le, A., Zhou, B., Shiu, H., Lee, C., Chang, W.: Numerical simulation and experimental validation of liquid water behaviors in a proton exchange membrane fuel cell cathode with serpentine channels. Journal of Power Sources 195(21), 7302-7315 (2010)

80. Le, A.D., Zhou, B.: Fundamental understanding of liquid water effects on the performance of a pemfc with serpentine-parallel channels. Electrochimica Acta 54(8), 2137-2154 (2009)

81. Lobato, J., Cañizares, P., Rodrigo, M., Pinar, F., Mena, E., Úbeda, D.: Three-dimensional model of a $50 \mathrm{~cm} 2$ high temperature pem fuel cell. study of the flow channel geometry influence. International Journal of Hydrogen Energy 35(11), 5510-5520 (2010)

82. Loubère, R., Maire, P.H., Shashkov, M., Breil, J., Galera, S.: Reale: A reconnection-based arbitrarylagrangian-eulerian method. Journal of Computational Physics 229(12), 4724-4761 (2010)

83. Luo, H., Baum, J.D., Löhner, R.: On the computation of multi-material flows using ale formulation. Journal of Computational Physics 194(1), 304-328 (2004)

84. Mancusi, E., Fontana, E., de Souza, A., de Souza, S.: Numerical study of two-phase flow patterns in the gas channel of pem fuel cells with tapered flow field design. International Journal of Hydrogen Energy 39(5), 22612273 (2014)

85. Manke, I., Hartnig, C., Grünerbel, M., Lehnert, W., Kardjilov, N., Haibel, A., Hilger, A., Banhart, J., Riesemeier, H.: Investigation of water evolution and transport in fuel cells with high resolution synchrotron x-ray radiography. Applied Physics Letters 90(17), 174,105 (2007)

86. Marti, J., Ryzhakov, P., Idelsohn, S., Oñate, E.: Combined eulerian-pfem approach for analysis of polymers in fire situations. International Journal for Numerical Methods in Engineering 92, 782-801 (2012)

87. Mazumder, S., Cole, J.V.: Rigorous 3-d mathematical modeling of pem fuel cells ii. model predictions with liquid water transport. Journal of the Electrochemical Society 150(11), A1510-A1517 (2003)

88. Mier-Torrecilla, M.: Numerical simulation of multi-fluid flows with the Particle Finite Element Method. Ph.D. thesis, Universitat Politècnica de Catalunya (2010)

89. Milne, A., Amirfazli, A.: Drop shedding by shear flow or hydrophilic to superhydrophobic surfaces. Langmuir 25(24), 14,155-14,164 (2009)

90. Minor, G.: Experimental study of water droplet flows in a model PEM fuel cell gas microchannel. Ph.D. thesis, University of Victoria (2007)

91. Mondal, B., Jiao, K., Li, X.: Three-dimensional simulation of water droplet movement in pem fuel cell flow channels with hydrophilic surfaces. International Journal of Energy Research 35(13), 1200-1212 (2011)

92. Mukaide, T., Mogi, S., Yamamoto, J., Morita, A., Koji, S., Takada, K., Uesugi, K., Kajiwara, K., Noma, T.: In situ observation of water distribution and behaviour in a polymer electrolyte fuel cell by synchrotron x-ray imaging. Journal of synchrotron radiation 15(4), 329$334(2008)$ 
93. O'Hayre, R., Cha, S.W., Prinz, F.B., Colella, W.: Fuel cell fundamentals. John Wiley \& Sons (2016)

94. Osher, S., Sethian, J.: Fronts propagating with curvature dependent speed: algorithms based on HamiltonJacobi formulations. Journal of Computational Physics 79, 12-49 (1988)

95. Pasaogullari, U., Wang, C.Y.: Two-phase modeling and flooding prediction of polymer electrolyte fuel cells. Journal of The Electrochemical Society 152(2), A380A390 (2005)

96. Polverino, P., Esposito, A., Pianese, C.: Experimental validation of a lumped model of single droplet deformation, oscillation and detachment on the gdl surface of a pem fuel cell. International Journal of Hydrogen Energy 38(21), 8934-8953 (2013)

97. Qin, C., Rensink, D., Hassanizadeh, S., Fell, S.: Direct simulation of liquid water dynamics in the gas channel of a polymer electrolyte fuel cell. Journal of The Electrochemical Society 159(4), B434-B443 (2012)

98. Qin, Y., Du, Q., Yin, Y., Jiao, K., Li, X.: Numerical investigation of water dynamics in a novel proton exchange membrane fuel cell flow channel. Journal of Power Sources 222, 150-160 (2013)

99. Qin, Y., Li, X., Du, Q., Yin, Y., Jiao, K.: Effect of wettability on water removal from the gas diffusion layer surface in a novel proton exchange membrane fuel cell flow channel. international journal of hydrogen energy 38(29), 12,879-12,885 (2013)

100. Qin, Y., Li, X., Jiao, K., Du, Q., Yin, Y.: Effective removal and transport of water in a pem fuel cell flow channel having a hydrophilic plate. Applied Energy 113, 116-126 (2014)

101. Quan, P., Lai, M.: Numerical study of water management in the air flow channel of a PEM fuel cell cathode. Journal of Power Sources 164, 222-237 (2007)

102. Quan, P., Lai, M.: Numerical simulation of two-phase water behavior in the cathode of an interdigitated proton exchange membrane fuel cell. Journal of Fuel Cell Science and Technology 7(1) (2010)

103. Quan, P., Zhou, B., Sobiesiak, A., Liu, Z.: Water behavior in serpentine micro-channel for proton exchange membrane fuel cell cathode. Journal of Power Sources 152, 131-145 (2005)

104. Raman, K., Mondal, B., Li, X.: Water droplet transport in single gas flow channel of pem fuel cell. International Journal of Advances in Thermal Sciences and Engineering 2(1), 27-33 (2011)

105. Rossi, R., Larese, A., Dadvand, P., Oñate, E.: An efficient edge-based level set finite element method for free surface flow problems. International Journal for Numerical Methods in Fluids 71(6), 687-716 (2013)

106. Ryzhakov, P., Oñate, E., Rossi, R., Idelsohn, S.: Lagrangian FE methods for coupled problems in fluid mechanics. CIMNE (2010)

107. Ryzhakov, P., Rossi, R., Oñate, E.: An algorithm for the simulation of thermally coupled low speed flow problems. International Journal for Numerical Methods in Fluids 70(1), 1-19 (2012)

108. Ryzhakov, P.B., Jarauta, A.: An embedded approach for immiscible multi-fluid problems. International Journal of Numerical Methods in Fluids 81, 357-376 (2015). DOI 10.1002/fld.4190

109. Ryzhakov, P.B., Jarauta, A., Secanell, M., Pons-Prats, J.: On the application of the PFEM to droplet dynamics modeling in fuel cells. Computational Particle Mechanics pp. 1-11 (2016)
110. Sabharwal, M., Pant, L.M., Putz, A., Susac, D., Jankovic, J., Secanell, M.: Analysis of catalyst layer microstructures: From imaging to performance. Fuel Cells 10(1002), 201600,008 (2016)

111. Saksono, P.H., Perić, D.: On finite element modelling of surface tension. Variational formulations and applications - Part I: Quasistatic problems. Computational Mechanics 38, 265-281 (2006)

112. Saksono, P.H., Perić, D.: On finite element modelling of surface tension. Variational formulations and applications - Part II: Dynamic problems. Computational Mechanics 38, 251-263 (2006)

113. Satija, R., Jacobson, D., Arif, M., Werner, S.: In situ neutron imaging technique for evaluation of water management systems in operating pem fuel cells. Journal of Power Sources 129, 238-245 (2004)

114. Sharaf, O.Z., Orhan, M.F.: An overview of fuel cell technology: Fundamentals and applications. Renewable and Sustainable Energy Reviews 32, 810-853 (2014)

115. Shirani, E., Masoomi, S.: Deformation of a droplet in a channel flow. Journal of fuel cell science and technology 5(4), 041,008 (2008)

116. Slikkerveer, P.J., Lohuizen, E.P.V., O'Brien, S.B.G.: An implicit surface tension algorithm for Picard solvers of surface-tension-dominated free and moving boundary problems. Int. Journal for Numerical Methods in Fluids 22, 851-865 (1996)

117. Suresh, P., Jayanti, S.: Effect of air flow on liquid water transport through a hydrophobic gas diffusion layer of a polymer electrolyte membrane fuel cell. International journal of hydrogen energy 35(13), 6872-6886 (2010)

118. Sussman, M., Ohta, M.: A stable and efficient method for treating surface tension in incompressible two-phase flow. SIAM Journal on Scientific Computing 31, 2447$2471(2009)$

119. Tezduyar, T.E.: Stabilized finite element formulations for incompressible flow computations. Advances in applied mechanics 28, 1-44 (1991)

120. Theodorakakos, A., Ous, T., Gavaises, M., Nouri, J., Nikolopoulos, N., Yanagihara, H.: Dynamics of water droplets detached from porous surfaces of relevance to PEM fuel cells. Journal of Colloid and Interface Science 300, 673-687 (2006)

121. Tuber, K., Pcza, D., Hebling, C.: Visualization of water buildup in the cathode of a transparent pem fuel cell. Journal of Power Sources 124, 403-414 (2003)

122. Uchiyama, T.: Ale finite element method for gas-liquid two-phase flow including moving boundary based on an incompressible two-fluid model. Nuclear engineering and design 205(1), 69-82 (2001)

123. Um, S., Wang, C.Y., Chen, K.: Computational fluid dynamics modeling of proton exchange membrane fuel cells. Journal of the Electrochemical society 147(12), 4485-4493 (2000)

124. Wang, M., Feindel, K.W., Bergens, S.H., Wasylishen, R.E.: In situ quantification of the in-plane water content in the nafion membrane of an operating polymerelectrolyte membrane fuel cell using $1 \mathrm{~h}$ micro-magnetic resonance imaging experiments. Journal of Power Sources 195, 7316-7322 (2010)

125. Wang, X., Zhou, B.: Liquid water flooding process in proton exchange membrane fuel cell cathode with straight parallel channels and porous layer. Journal of Power Sources 196(4), 1776-1794 (2011)

126. Wang, Z., Wang, C., Chen, K.: Two-phase flow and transport in the air cathode of proton exchange membrane fuel cells. Journal of Power Sources 94(1), 40-50 (2001) 
127. Whitaker, S.: Flow in porous media i: A theoretical derivation of darcy's law. Transport in porous media 1(1), 3-25 (1986)

128. Wörner, M.: Numerical modeling of multiphase flows in microfluidics and micro process engineering: a review of methods and applications. Microfluidics and nanofluidics 12(6), 841-886 (2012)

129. Wu, T., Djilali, N.: Experimental investigation of water droplet emergence in a model polymer electrolyte membrane fuel cell microchannel. Journal of Power Sources 208, 248-256 (2012)

130. Yang, X.G., Zhang, F.Y., Lubawy, A.L., Wang, C.Y.: Visualization of liquid water transport in a pefc. Electrochemical and Solid-State Letters 7(11), A408-A411 (2004)

131. You, L., Liu, H.: A two-phase flow and transport model for the cathode of pem fuel cells. International journal of heat and mass transfer 45(11), 2277-2287 (2002)

132. Zhan, Z., Wang, C., Fu, W., Pan, M.: Visualization of water transport in a transparent pemfc. International Journal of Hydrogen Energy 37, 1094-1105 (2012)

133. Zhan, Z., Xiao, J., Pan, M., Yuan, R.: Characteristics of droplet and film water motion in the flow channels of polymer electrolyte membrane fuel cells. Journal of Power Sources 160(1), 1-9 (2006)

134. Zhang, F.Y., Yang, X.G., Wang, C.Y.: Liquid water removal from a polymer electrolyte fuel cell. Journal of The Electrochemical Society 153(2), A225-A232 (2006)

135. Zhou, J., Putz, A., Secanell, M.: A mixed wettability pore size distribution based mathematical model for analyzing two-phase flow in porous electrodes i. mathematical model. Journal of The Electrochemical Society 164(6), F530-F539 (2017)

136. Zhu, X., Liao, Q., Sui, P., Djilali, N.: Numerical investigation of water droplet dynamics in a low-temperature fuel cell microchannel: Effect of channel geometry. Journal of Power Sources 195, 801-812 (2010)

137. Zhu, X., Sui, P., Djilali, N.: Numerical simulation of emergence of a water droplet from a pore into a microchannel gas stream. Microfluid Nanofluid 4, 543-555 (2008)

138. Zhu, X., Sui, P., Djilali, N.: Three-dimensional numerical simulations of water droplet dynamics in a PEMFC gas channel. Journal of Power Sources 181, 101-115 (2008)

139. Zhu, X., Sui, P., Djilali, N., Liao, Q.: Dynamics of emerging water droplet subjected to sidewall with different wettabilities in a fuel cell cathode channel. Fuel Cells 11(3), 404-412 (2011)

140. Zhu, X., Sui, P.C., Djilali, N.: Dynamic behaviour of liquid water emerging from a GDL pore into a PEMFC gas flow channel. Journal of Power Sources 172, 287-295 (2007) 